\title{
PRINCIPLES OF SEISMIC HOLOGRAPHY FOR DIAGNOSTICS OF THE SHALLOW SUBPHOTOSPHERE
}

\author{
Charles Lindsey and D. C. Braun \\ NorthWest Research Associates, Colorado-Research Associates Division, Boulder, CO 80301; \\ clindsey@cora.nwra.com,dbraun@cora.nwra.com \\ Received 2003 December 29; accepted 2004 July 6
}

\begin{abstract}
We develop the wave-mechanical formalism for phase-correlation computational seismic holography of the shallow subphotosphere under the plane-parallel approximation and apply it to helioseismic Doppler observations from the Michelson Doppler Imager on the $\mathrm{SOHO}$ spacecraft of both the quiet Sun and active regions. We compare holographic signatures computed wave-mechanically with similar signatures computed under the widely used eikonal approximation. The major difference between the hydromechanical and eikonal computations can be expressed in terms of acoustic dispersion effects within a few $\mathrm{Mm}$ of the solar surface. With an appropriate account for dispersion, the eikonal computations are remarkably accurate over a broad range of practical applications. A major imposition that confronts local diagnostics of the shallow subphotosphere is a phenomenon we call "ghost signatures," artifacts introduced by a local ambiguity in the origin of the waves that give rise to the helioseismic signatures observed in the photosphere. Phase-correlation holographic signatures of the shallow subphotospheres of active regions are predominated by strong, stochastic phase shifts associated with magnetic fields at the solar surface. These introduce effects similar to those of an optical showerglass, significantly impairing the coherence of waves impinging into the magnetic photosphere from beneath, smearing the holographic signatures of possible subphotospheric anomalies.
\end{abstract}

Subject headings: Sun: activity — Sun: helioseismology — sunspots

\section{INTRODUCTION}

Computational seismic holography of helioseismic observations has rendered a remarkable array of diagnostic utilities for solar research. These have led to the following developments:

1. the recognition of extended seismic anomalies surrounding sunspots, called "acoustic moats" (Lindsey \& Braun 1998; Braun et al. 1998; Braun \& Lindsey 2000a, 2000b);

2 . the discovery of regions of anomalous high-frequency seismic emission surrounding large active regions, called "acoustic glories" (Braun \& Lindsey 1999; Donea et al. 1999);

3. seismic images of solar flares (Donea et al. 2000); and

4. synoptic images of large active regions on the far surface of the Sun (Lindsey \& Braun 2000a; Braun \& Lindsey 2001).

The diagnostic potential of helioseismic holography encompasses a broad range of practical applications basic to local helioseismology. The major promise lies in solar interior diagnostics. Given the appropriate helioseismic observations of the solar surface, it offers diffraction-limited discrimination of local solar interior anomalies at any depth beneath the Sun's surface.

Diagnostics of the shallow subphotosphere are of critical importance to local helioseismology. A great deal of interesting solar acoustics occurs in the shallow subphotosphere. Moreover, we have to look through the shallow subphotosphere to see into the deeper interior.

Most of the waves visible on the solar surface are highdegree modes (meaning that their surface ripples have a high wavenumber, $k$, or alternatively a short wavelength, $2 \pi / k$ ). These waves penetrate only a relatively short distance beneath the photosphere. In fact, it is these high- $k$ waves that offer the finest spatial discrimination. Diagnostics of the shallow subphotosphere are crucial to an understanding of the physics of active regions. The same applies to the supergranulation and other photospheric features that we can see directly.

The computational requirements of seismic holography vary widely depending on the specific applications. Since only relatively low- $k$ waves penetrate deep into the solar interior, diagnostics of the deep interior or far surface of the Sun can be accomplished with relatively coarse spatial sampling. In this case, the most practical approach is to filter the observations at an early stage for just the low- $k$ modes, reducing the computational task proportionately. For diagnostics of the shallow subphotosphere, high- $k$ modes become important, greatly increasing the sampling requirements in all spatial dimensions. The purpose of this paper is to develop a wave-mechanical formalism for seismic holography of the shallow subphotosphere that addresses the computational requirements in a practical way. The most basic concepts, including those of computational economy, were already reviewed by Lindsey \& Braun (2000b). The computational task for the shallow subphotosphere can be greatly reduced by a judicious application of the plane-parallel approximation. This will be the context of this paper.

We will use a fair amount of optical terminology in this paper. When we use the term "radiation," for example, the meaning will be "acoustic radiation," not electromagnetic unless this qualification is stated. Nearly all work in helioseismic holography to date has relied on a formalism known in optics as the "eikonal approximation" (see $\S 4$ of Lindsey \& Braun 1997 and $\S 8.1-8.2$ of Lindsey \& Braun 2000b). The eikonal approximation turns out to be excellent for all of the work cited above. There are, however, applications in the shallow subphotosphere in which the eikonal approximation opens the door to possibly significant errors. Some of these were pointed out by Barnes \& Cally (2001). Others are described below. This paper addresses those liabilities by working out the wave-mechanics 
of computational seismic holography for the shallow solar subphotosphere formally without reliance on the eikonal approximation. Comparisons of holographic seismic images computed according to this formalism and those based on the eikonal approximation show why the eikonal approximation, when supplemented with appropriate corrections for dispersion, is a remarkably accurate utility for imaging the solar photosphere and subphotosphere when based on waves that penetrate a few $\mathrm{Mm}$ or more beneath the solar surface. We apply phase-correlation seismic holography to the quiet Sun and an active region and examine two of the more imposing artifacts that confront shallow subphotospheric diagnostics. The first of these is "ghost signatures," largely caused by ambiguity in wave travel directions. The second is the effects of large phase and amplitude perturbations introduced by surface magnetic fields.

We will begin with a review of the general principles of computational seismic holography. We will proceed with the formal development of wave-mechanics in a plane-parallel acoustic medium stratified by gravity, for which the sound speed increases with depth, as in the solar subphotosphere. Finally, we will apply the formalism to helioseismic observations to compare holographic signatures computed under the eikonal approximation with images computed according to the principles of formal wave-mechanics.

\section{REVIEW}

\subsection{Basic Principles}

Basic principles of helioseismic holography were described and illustrated by Lindsey \& Braun (2000b). The heart of the diagnostic is the computational extrapolation of acoustic disturbances observed at the solar surface into the solar interior assuming an interior medium devoid of acoustic anomalies. Computational seismic holography can be regarded as the direct extension to solar seismology of basic elements of a diagnostic in geoseismology called "seismic migration" (Schneider 1978; Berkhout 1985). The theory and applications of seismic migration were extensively developed in the $1970 \mathrm{~s}$ (Claerbout 1970; Hilterman 1970; Claerbout 1971; Claerbout \& Doherty 1972). However, significant elements of the theory (e.g., Musgrave 1961) go back years earlier, proceeding to some degree in parallel with the practical development of optical holography. The concept was recognized for helioseismic applications in terms of its analogy to optical holography by Roddier (1975), an association adopted by Lindsey \& Braun (1990, 1997, 2000b) and Braun et al. (1998) owing to their greater familiarity with elementary optics and the applicability of a broad range of familiar optical terminology and other formalism.

In rough generality, the computational basis of seismic holography is the partial reconstruction, $H_{ \pm}$, of the acoustic field, $\psi$, beneath the solar surface by phase-coherent acoustic progression or regression some distance into the solar interior, based on observations of $\psi$ at the surface, as prescribed by a nominal acoustic model that is devoid of underlying acoustic anomalies. This reconstruction for waves at a particular angular frequency, $\omega$, is conveniently expressed in terms of a surface integral over some region, $\mathcal{P}$, of the form

$$
H_{ \pm}^{\mathcal{P}}(\boldsymbol{R}, \omega)=\int_{\mathcal{P}} d^{2} \boldsymbol{R}^{\prime} G_{ \pm}\left(\boldsymbol{R}, \boldsymbol{R}^{\prime}, \omega\right) \psi\left(\boldsymbol{R}^{\prime}, \omega\right)
$$

where the "Green's functions," $G_{-}$and $G_{+}$, represent the nominal acoustic model as complex propagators whose basic purpose is to evolve the acoustic field, forward and backward in time, respectively, from the surface at point $\boldsymbol{R}^{\prime}$ to a submerged "focal point," $\boldsymbol{R}$. The time-reverse and time-forward Green's functions are related to each other by

$$
G_{+}\left(\boldsymbol{R}, \boldsymbol{R}^{\prime}, \omega\right)=G_{-}^{*}\left(\boldsymbol{R}, \boldsymbol{R}^{\prime}, \omega\right)
$$

We call the forward progression of the acoustic field to $\boldsymbol{R}$ the "coherent acoustic ingression," and its regression, backward in time, the "coherent acoustic egression." The region, $\mathcal{P}$, over which the integral is computed is called the "pupil" of the computation. We will usually use the term "progression" to refer to the computational evolution, $H_{-}$, of the acoustic field forward in time and "regression" to refer to $H_{+}$, the evolution backward. However, we will sometimes defer to "progression" to express the generality, even when the direction of the extrapolation could be backward in time.

For computational purposes, it is helpful to distinguish between the depth, $z$, represented by $\boldsymbol{R}$ and the horizontal location, $\boldsymbol{r}$, of the surface point directly beneath which $\boldsymbol{R}$ lies:

$$
\boldsymbol{R} \equiv(\boldsymbol{r}, z)
$$

This allows us to take computational advantage of the horizontal invariance of acoustics in a stratified atmosphere. $G_{ \pm}$ can be expressed as a function of $\left|\boldsymbol{r}-\boldsymbol{r}^{\prime}\right|$ and $z$, covering a two-dimensional manifold in place of the six dimensions occupied by $\left(\boldsymbol{R}, \boldsymbol{R}^{\prime}\right)$. Thus,

$$
H_{ \pm}^{\mathcal{P}}(\boldsymbol{r}, z, \omega)=\int_{\mathcal{P}} d^{2} \boldsymbol{r}^{\prime} G_{ \pm}\left(\left|\boldsymbol{r}-\boldsymbol{r}^{\prime}\right|, z, \omega\right) \psi\left(\boldsymbol{r}^{\prime}, \omega\right) .
$$

In our applications, the pupil is invariant with respect to the focus and can therefore be expressed as part of $G_{ \pm}$in terms of a multiplicative step function, $U\left(\boldsymbol{r}-\boldsymbol{r}^{\prime}\right)$ that simply truncates $G_{ \pm}$at the pupil boundary. In that case the integral in equation (4) is a simple convolution. The spatial Fourier transform, $\hat{H}_{ \pm}$, of $H_{ \pm}$over the surface therefore reduces to a simple product (Lindsey \& Braun 2000b), greatly expediting the computation:

$$
\hat{H}_{ \pm}(\boldsymbol{k}, z, \omega)=\hat{G}_{ \pm}(\boldsymbol{k}, z, \omega) \hat{\psi}(\boldsymbol{k}, \omega)
$$

(Lindsey \& Braun 2000b). Here the wavevector $\boldsymbol{k}$, like $\boldsymbol{r}$, lies in the horizontal plane, with $\hat{G}_{ \pm}(\boldsymbol{k}, z, \omega)$ and $\hat{\psi}(\boldsymbol{k}, \omega)$ representing the two-dimensional spatial Fourier transforms of $G_{ \pm}\left(\left|\boldsymbol{r}^{\prime}\right|, z, \omega\right)$ and $\psi\left(\boldsymbol{r}^{\prime}, \omega\right)$, respectively.

When $H_{+}$or $H_{-}$is computed over a surface, we call this manifold the "focal surface." This will generally be a surface of constant depth, to which we will apply the term "focal plane," by benefit of the plane-parallel approximation. Aberrations introduced by the plane-parallel approximation were briefly described in $\S 3$ of Lindsey \& Braun (2000b). Beginning with equation (5), we will omit the " $\mathcal{P}$ " superscript from $H_{ \pm}$until it is first needed, in $\S 5.4$.

Figure 1 illustrates how the relationship between the horizontal extent of the pupil and the depth of the focus determines the "vantage" of the reconstruction (see $\S 4$ of Lindsey $\&$ Braun 2000b). This study will make extensive use of the subjacent vantage (Fig. $1 b$ ) and the lateral vantage (Figs. $1 c$ and $1 d)$. The subjacent vantage is useful for control work focused on the solar surface. The lateral vantage is useful phase-correlation diagnostics of substantially submerged focal planes. Most holographic applications published to date have been computed from the subjacent vantage, with a focus at or near the surface. 

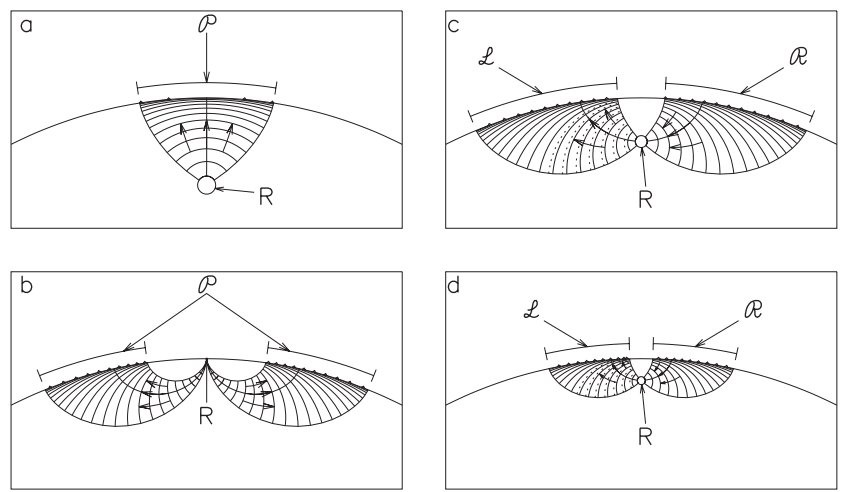

FIG. 1.-Wave front diagrams illustrating holographic extrapolations from $(a)$ a superjacent vantage, $(b)$ a subjacent vantage, and $(c, d)$ lateral vantages, for which the horizontal extent of the pupil expands rapidly with increasing focal depth.

\subsection{Incompleteness}

While helioseismic observations are highly informative, they are generally far from sufficient for a full reconstruction of the interior acoustic field, even for a medium devoid of acoustic anomalies. A medium characterized by a simple scalar amplitude, $\psi$, satisfying the familiar wave equation

$$
\frac{\partial^{2}}{\partial t^{2}} \psi-c^{2} \nabla^{2} \psi=0
$$

in three dimensions with a uniform sound speed, $c$, facilitates a general assessment of wave-mechanical incompleteness and its consequences. Wave-mechanical reconstruction of $\psi$ into the interior, $\mathcal{R}$, of a closed surface, $\partial \mathcal{R}$, is accomplished forward in time by the Kirchhoff integral (see Born \& Wolf 1975; Schneider 1978),

$$
\begin{aligned}
\psi(\boldsymbol{R}, \omega)= & \int_{\partial \mathcal{R}} d^{2} \boldsymbol{R}^{\prime}\left[\psi\left(\boldsymbol{R}^{\prime}, \omega\right) \frac{\partial}{\partial n^{\prime}} G_{-}^{M}\left(\boldsymbol{R}, \boldsymbol{R}^{\prime}, \omega\right)\right. \\
& \left.-G_{-}^{M}\left(\boldsymbol{R}, \boldsymbol{R}^{\prime}, \omega\right) \frac{\partial}{\partial n^{\prime}} \psi\left(\boldsymbol{R}^{\prime}, \omega\right)\right],
\end{aligned}
$$

if $\psi$ is secured over the entirety of $\partial \mathcal{R}$ as well as its derivative, $\partial \psi / \partial n^{\prime}$, normal to $\partial \mathcal{R}$. While helioseismic observations in the present epoch may accommodate us with credible facsimiles of either $\psi$ or $\partial \psi / \partial n^{\prime}$ alone over a substantial pupil, $\mathcal{P}$, on the solar surface facing Earth, these do not nearly cover the entire solar surface and generally do not provide us with both over any substantial region. As in most optical applications, holographic progressions assume the missing field value is zero on $\partial \mathcal{R}$, where it is not known. If the observations provide us with a map of $\psi$ in surface region $\mathcal{P}$ on $\partial \mathcal{R}$, we accomplish a partial reconstruction, $H_{-}$, of $\psi$ beneath the surface forward in time by letting

$$
G_{-}\left(\left|\boldsymbol{R}-\boldsymbol{R}^{\prime}\right|, z, \omega\right) \equiv \frac{\partial}{\partial n^{\prime}} G_{-}^{M}\left(\boldsymbol{R}, \boldsymbol{R}^{\prime}, \omega\right)
$$

in equation (4). The result of restricting the pupil, $\mathcal{P}$, to a limited region is familiar in elementary optics as smearing known by the term "diffraction." As in standard optics, the smaller the pupil, the greater the smearing and the poorer the spatial discrimination. On the other hand, the omission of either of the two terms that comprise the Kirchhoff integrand results in a confusion that is somewhat more subtle, which we will call "directional ambiguity." This imposition, certain aspects of which were addressed by Skartlien (2001, 2002), is familiar in geoseismic diagnostics (Claerbout \& Doherty 1972; Castle 1982) as well as in optical holography, where it gives rise to unintended mirror-like images of the object. In computational seismic holography these appear as noisy, outof-focus artifacts we call "ghost signatures," to be discussed at length in $\S \S 5.3,5.7$, and 7.3.

\subsection{Phase-Correlation Holography}

The general basis of phase-correlation holography is the correlation,

$$
C(\boldsymbol{R})=\left\langle H_{+}(\boldsymbol{R}, \omega) H_{-}^{*}(\boldsymbol{R}, \omega)\right\rangle_{\Delta \omega},
$$

between the egression and ingression at a common focal point (Braun \& Lindsey 2000b), wherein the angular brackets express the mean of the contents over a frequency range represented by $\Delta \omega$. In a complete reconstruction of the acoustic field in a medium devoid of anomalies, the egression, $H_{+}(\boldsymbol{R}, \omega)$, and ingression, $H_{-}(\boldsymbol{R}, \omega)$, would be equal. In practice, incompleteness allows $H_{-}$and $H_{+}$to differ substantially, even in an ideal medium devoid of scatterers. Nevertheless, given that the computations are made over appropriate respective pupils, $\mathcal{P}_{ \pm}$, the ingression and egression at the same focal point, $\boldsymbol{R}$, generally have a strong statistical correlation.

$C(\boldsymbol{R})$ is influenced by acoustic anomalies in the medium. Of particular interest is the response of $C(\boldsymbol{R})$ to elastic scatterers, such as refractors and flows, which are not generally visible to simple acoustic power holography (Lindsey \& Braun 1997, 2000a). Local refractors and Doppler scatterers generally shift the phase of $C(\boldsymbol{R})$ (see eq. [9] of Lindsey \& Braun 2000a). This signature strongly favors local anomalies occupying the immediate neighborhood of the focal point, $\boldsymbol{R}$.

\section{THE EIKONAL APPROXIMATION}

The eikonal approximation has been remarkably useful in the early application of computational seismic holography, giving us diffraction-limited images of acoustic moats, acoustic glories, solar flares, and active regions on the Sun's far surface. The purpose of the discussion that follows is to review the basic principles of the eikonal approximation and to clarify its limitations. A more detailed discussion of the eikonal approximation and its practical application to computational holography was described by Lindsey \& Braun (2000b). In the eikonal approximation, the Green's function, $G_{ \pm}$, is supposed to have the form

$G_{ \pm}\left(\left|\boldsymbol{r}-\boldsymbol{r}^{\prime}\right|, z, \omega\right)=f\left(\left|\boldsymbol{r}-\boldsymbol{r}^{\prime}\right|, z, \omega\right) e^{ \pm i S\left(\left|\boldsymbol{r}-\boldsymbol{r}^{\prime}\right|, z, \omega\right)}$,

where $f$ is a real, positive modulus, and the eikonal, $S$, expresses the progression of phase along the path, $\Gamma$, of minimum travel time from $\left(\boldsymbol{r}^{\prime}, 0\right)$ to $(\boldsymbol{r}, z)$ :

$$
S\left(\left|\boldsymbol{r}-\boldsymbol{r}^{\prime}\right|, z, \omega\right)=\int_{\Gamma} \frac{\omega}{c} d s
$$

with $d s$ expressing the differential element of path length along $\Gamma$. The condition of minimum travel time is satisfied by the requirement that $\Gamma$ obey Snell's law of refraction:

$$
\frac{d r}{d s}=\frac{c}{c_{\mathrm{ph}}} \sin \theta_{\mathrm{ph}}
$$


where $d r$ represents the horizontal progression along $\Gamma$ of path element $d s, c_{\mathrm{ph}}$ is the speed of sound at the photosphere (or any desired reference depth), and $\theta_{\mathrm{ph}}$ is the tangent angle of $\Gamma$ from the vertical at the photosphere. The modulus, $f$, in equation (10) can be approximated by the "intensity law of geometrical optics" based on considerations of acoustic flux conservation (see $\S 8.1$ of Lindsey \& Braun 2000b).

By treating plane waves in a quasi-uniform medium, ${ }^{1}$ with the imposition that the vertical component of the acoustic flux is independent of height, it is possible to derive an eikonal analog for the Fourier transform of $G_{ \pm}$:

$$
\hat{G}_{ \pm}(k, z, \omega)=\tilde{f}(k, z, \omega) e^{ \pm i \tilde{S}(k, z, \omega)},
$$

with

$$
\tilde{S}(k, z, \omega)=\int\left[\frac{\omega^{2}}{c^{2}(z)}-k^{2}\right]^{1 / 2} d z
$$

and

$$
\tilde{f}^{2}(k, z, \omega)=\frac{\sigma}{c}\left[1-\frac{k^{2} c^{2}(z)}{\omega^{2}}\right]^{-1 / 2},
$$

where $\sigma$ is a constant that normalizes $\tilde{f}$ to unity at the surface of the medium.

The modulus, $\tilde{f}$, of $\hat{G}_{ \pm}$, experiences a singularity at the depth for which $c=\omega / k$, the lower turning point of the ray path, at which refraction turns the wave trajectory back toward the surface of the medium. In a formal hydromechanical treatment, the singularity will be seen to relax to an analytic diffraction fringe.

\section{WAVE-MECHANICS OF SEISMIC HOLOGRAPHY IN A PLANE-PARALLEL GRAVITATIONALLY STRATIFIED MEDIUM}

The most widely used helioseismic observations available today are Doppler observations, such as those made by the Michelson Doppler Imager (MDI) aboard the Solar Heliospheric Observatory (SOHO) spacecraft and by the Global Oscillations Network Group (GONG). Doppler observations near solar disk center give us accurate maps of the vertical component of the motion of the solar medium due to acoustic disturbances. This suggests that we undertake a careful account of acoustic disturbances in the solar interior in terms of the vertical displacement of the solar interior medium. The acoustic model we adapt for this purpose, then, characterizes hydromechanical disturbances in a plane-parallel acoustic medium stratified by gravity in terms of a vector field $\boldsymbol{\xi}(\boldsymbol{r}, z, t)$, that represents the acoustic displacement of the solar medium in three spatial dimensions, at $(\boldsymbol{r}, z)$ and time $t$. We represent the foregoing model with an adaptation of equation (14.25) of Unno et al. (1989), whereby $\boldsymbol{\xi}$ evolves temporally by a linear wave equation of the form

$$
\frac{\partial^{2} \boldsymbol{\xi}}{\partial t^{2}}=\frac{1}{\rho_{0}} \nabla\left(\rho_{0} c_{0}^{2} \nabla \cdot \boldsymbol{\xi}\right)-\boldsymbol{g} \nabla \cdot \boldsymbol{\xi}+\nabla(\boldsymbol{g} \cdot \boldsymbol{\xi}) .
$$

\footnotetext{
${ }^{1}$ By "quasi-uniform" we mean a medium in which the sound speed changes by a relatively small fraction of itself in a nominal wavelength. Thus,
}

$$
|\nabla c| \ll \nu
$$

Here $g$ is the local gravity vector, which is assumed unchanged by acoustic perturbations, and we otherwise identify physical parameters characterizing properties of the unperturbed medium by appending the subscript " 0 ." Thus, the gradients, $\nabla$, are computed with respect to $\boldsymbol{R}$ (see eq. [3]), $\rho_{0}(z)$ is the density of the unperturbed solar medium, $c_{0}(z)$ is the sound speed, and $\rho_{0} c_{0}^{2}$ expresses Young's modulus for local adiabatic compression of the initially unperturbed medium. The perturbation in pressure is

$$
p=-\rho_{0} c_{0}^{2} \nabla \cdot \boldsymbol{\xi}
$$

Equation (16) prescribes how acoustic disturbances propagate through the interior of the acoustic model once they are somehow introduced. We now postulate a model consisting of such a medium initially undisturbed and bounded by an upper surface, $\mathcal{S}$, that is initially flat and referenced to depth $z=0$. Beginning at some arbitrary time, $t=0$, we can impose onto $\mathcal{S}$ any vertical perturbation we choose that can be expressed in terms of a continuous function, $\xi_{z}(\boldsymbol{r}, 0, t)$, of position, $\boldsymbol{r}$, on the surface and time, $t$. Computational seismic holography applied to observations of vertical surface motion in the planeparallel approximation in this paper will be based largely on the following principle, tentatively stated as a proposition.

ASSERTION 1. If we drive the upper surface of the foregoing model, initially undisturbed, depressing it vertically as a function of surface location and time in such a fashion as described above, then a disturbance will propagate into the model interior in an unambiguous, well-defined way.

At the same time we impose another assertion.

AsSERTION 2. The wave equation (eq. [16]) can be solved unambiguously for $\boldsymbol{\xi}$ throughout $\mathcal{R}$ given both the vertical displacement, $\xi_{z}$ and the pressure perturbation, $p$, on $\mathcal{S}$ over an appropriate duration.

Critical to Assertion 1, that the disturbance can be determined unambiguously from only $\xi_{z}$ at the surface with no knowledge of $p$, is the condition that the medium was initially undisturbed. This secures that the disturbance that results from driving $\xi_{z}$ at the surface is purely downward-propagating. It is the possibility of upward-propagating waves, such as from some submerged source, that introduces an ambiguity if only $\xi_{z}$ at the surface is known. ${ }^{2}$

Assertions 1 and 2 address the issue of incompleteness in the discussion of computational holography based on Doppler observations in the plane-parallel approximation. That Assertion 2 does not contradict Assertion 1 is a result of the imposition before the latter that the surface disturbance, $\xi_{z}(\boldsymbol{r}, 0, t)$, was the sole source of the underlying interior acoustic disturbances introduced therein.

The proof of Assertion 2 is straightforward and is derived in the next section. Assertion 1 follows readily from that discussion. We will adapt a standard hydromechanical formalism to express general solutions of the wave equation (16) in terms of $\xi_{z}$ and $p$ at the model surface, $\mathcal{S}$. We will then examine the relationship between $\xi_{z}$ and $p$ for purely upward- and purely downward-propagating waves to discriminate between the two. For computations of seismic progressions, time-forward extrapolations of acoustic disturbances into the solar interior,

\footnotetext{
${ }^{2}$ For a concrete illustration, imagine $\mathcal{S}$ being held firmly rigid, i.e., $\xi_{z}(\boldsymbol{r}, 0, t)=0$, while an upward-propagating wave from a submerged source impinges onto it. The upcoming wave would reflect off of $\mathcal{S}$ and back into the model interior causing pressure perturbations, $p$, at $\mathcal{S}$ but no displacement, $\xi_{z}$. The same null surface motion could just as well characterize a medium with no interior acoustic disturbances at all. This ambiguity disappears if upwardpropagating waves are disallowed.
} 
we will accomplish completeness artificially by imposing the following.

Assumption 1. All surface disturbances that appear in the Doppler observations represent downward-propagating waves, as if emanating from a surface source by driving the surface as it is seen to move. We suppose that these waves propagate downward, into the solar interior, encountering no acoustic anomalies until their arrival at the focal plane.

For computations of seismic regressions, time-reverse extrapolations of acoustic disturbances into the solar interior, we will accomplish the same with the opposite assumption.

Assumption 2. All observed surface disturbances are manifestations of waves emanating from the focal plane, at depth $z$ in the solar interior, that have arrived without encountering any acoustic anomalies between the focal plane and the surface.

It may appear that Assumptions 1 and 2 cannot both generally be correct, indeed that each diametrically contradicts the other. In fact, below $\sim 5 \mathrm{mHz}$, the solar surface acts as a strong, essentially specular reflector. This provides every upcoming wave a down-going echo, such that the surface disturbance becomes simultaneously the signature of both. In that particular case, Assumptions 1 and 2 can both be accurate, subject to qualifications that have to do with the phase relationship between the surface signature and the individual arriving and departing wave components. Above $\sim 5 \mathrm{mHz}$ the solar surface is a poor specular reflector, and helioseismic observations appear to be incomplete by their nature. In general, Assumptions 1 and 2 introduce artifacts, which we will discuss in $\S 5.8$.

\section{PROPAGATION OF SURFACE DISTURBANCES IN A PLANE-PARALLEL ACOUSTIC MEDIUM}

\subsection{The Eigenwaves}

In this section, we will address the general problem of extrapolating disturbances in the vertical displacement, $\xi_{z}$, viewed at the surface of a gravitationally stratified acoustic medium in the plane-parallel approximation to any depth beneath the surface, under the assumption that the surface is the sole source, or alternatively the sole sink, of the disturbance. We will first adapt a standard formalism for solving the wave equation (16), based on boundary specifications of both vertical displacement, $\xi_{z}$, and pressure perturbation, $p$. We will then apply standard analytical tools to discriminate between downward- and upward-propagating disturbances, selecting in favor of one or the other as the application requires to eliminate the ambiguity caused by the lack of knowledge of $p$ over $\mathcal{S}$.

Because the wave equation for the quiet Sun is temporally and horizontally invariant in the plane-parallel approximation, its solutions in any horizontal plane can be expressed in terms of plane waves:

$$
\boldsymbol{\xi}(\boldsymbol{r}, z, t)=\hat{\xi}(k, z, \omega) e^{i(\boldsymbol{k} \cdot \boldsymbol{r}-\omega t)} .
$$

We can therefore implement equation (5) in the Fourier domain in terms of the field $\xi_{z}$ by having $\hat{\psi}$ and $\hat{G}_{ \pm}$in equation (5) represented in the following terms:

$$
\hat{\psi}(\boldsymbol{k}, 0, \omega) \equiv \hat{\xi}_{z}(\boldsymbol{k}, 0, \omega)
$$

and

$$
\hat{G}_{ \pm}(\boldsymbol{k}, z, \omega) \equiv \hat{\xi}_{z \pm}(\boldsymbol{k}, z, \omega),
$$

where the "radial functions," $\hat{\xi}_{z+}$ and $\hat{\xi}_{z-}$, represent the vertical components of the downward- and upward-propagating solutions, $\hat{\xi}_{+}$and $\hat{\xi}_{-}$, respectively, of the wave equation such that

$$
\hat{\xi}_{z \pm}(k, 0, \omega)=1 .
$$

To determine the solutions, $\hat{\xi}_{z}(k, z, \omega)$, of the wave equation for general purposes, we take an approach essentially parallel to the formalism of Thomas et al. (1971), which expresses $\xi_{z}$ and pressure, $p$, in terms of a first-order differential equation in $z$. Using equation (17) to express $\nabla \cdot \boldsymbol{\xi}$ in terms of pressure, $p$, and replacing the operator $\partial / \partial t$ with $-i \omega$, the wave equation (16) becomes

$$
-\omega^{2} \boldsymbol{\xi}=-\frac{1}{\rho_{0}} \nabla p+\frac{\boldsymbol{g}}{c_{0}^{2}} \frac{p}{\rho_{0}}+(\nabla \boldsymbol{g}) \cdot \boldsymbol{\xi}+(\nabla \boldsymbol{\xi}) \cdot \boldsymbol{g} .
$$

We introduce the symbols $\boldsymbol{\xi}_{\perp}$ and $\xi_{z}$ to distinguish between the horizontal and vertical components of $\xi$ :

$$
\boldsymbol{\xi}=\left(\boldsymbol{\xi}_{\perp}, \xi_{z}\right),
$$

and express the vertical and horizontal components of the wave equation itself in terms of these:

$$
-\omega^{2} \xi_{z}=-\frac{1}{\rho_{0}} \frac{\partial p}{\partial z}+\frac{g}{c_{0}^{2}} \frac{p}{\rho_{0}}+\frac{d g}{d z} \xi_{z}+g \frac{d \xi_{z}}{d z},
$$

and

$$
-\omega^{2} \boldsymbol{\xi}_{\perp}=-i \boldsymbol{k} \frac{p}{\rho_{0}}+i \boldsymbol{k g} \xi_{z} .
$$

Equation (17) similarly leads to

$$
-\frac{p}{\rho_{0} c_{0}^{2}}=\frac{\partial \xi_{z}}{\partial z}+i \boldsymbol{k} \cdot \boldsymbol{\xi}_{\perp} .
$$

Taking the dot product of both sides of equation (25) with $i \boldsymbol{k}$ leads to an expression for $i \boldsymbol{k} \cdot \boldsymbol{\xi}_{\perp}$ in terms of $p$ and $\xi_{z}$ :

$$
\omega^{2} i \boldsymbol{k} \cdot \boldsymbol{\xi}_{\perp}=k^{2}\left(g \xi_{z}-\frac{p}{\rho_{0}}\right),
$$

to replace $i \boldsymbol{k} \cdot \boldsymbol{\xi}_{\perp}$ in equation (26). This leads to the following expression for $\partial \xi_{z} / \partial z$ in terms of $\xi_{z}$ and $p$ :

$$
\omega^{2} \frac{\partial \xi_{z}}{\partial z}=\left(k^{2}-\frac{\omega^{2}}{c_{0}^{2}}\right) \frac{p}{\rho_{0}}-k^{2} g \xi_{z} .
$$

Replacing $\partial \xi_{z} / \partial z$ in equation (24) with the above then renders an equation for $\partial p / \partial z$ in terms of $\xi_{z}$ and $p$ :

$$
\omega^{2} \frac{\partial p}{\partial z}=k^{2} g p+\rho_{0}\left(\omega^{4}+\omega^{2} \frac{d g}{d z}-k^{2} g^{2}\right) \xi_{z} .
$$

Equations (28) and (29) are readily expressed in matrix format:

$$
\omega^{2} \frac{\partial}{\partial z}\left(\begin{array}{c}
\xi_{z} \\
p
\end{array}\right)=\boldsymbol{M}_{0}(z)\left(\begin{array}{c}
\xi_{z} \\
p
\end{array}\right)
$$

with

$$
\boldsymbol{M}_{0}=\left[\begin{array}{cc}
-k^{2} g & \left(k^{2}-\omega^{2} / c_{0}^{2}\right) / \rho_{0} \\
\rho_{0}\left(\omega^{4}-\omega^{2} \omega_{T}^{2}-k^{2} g^{2}\right) & k^{2} g
\end{array}\right],
$$


where $\omega_{T}$, defined by

$$
\omega_{T}^{2} \equiv-\frac{d g}{d z}
$$

represents the tidal effect of the Sun's gravitational gradient, whose imaginary value of $(0.88 \mathrm{mHz}) i$ at the solar surface is sufficient to qualify it as a computational irritant.

Given $\xi_{z}$ and $p$ at any depth, $z_{\text {ref }}$, we can extrapolate these for nonzero $\omega^{2}$ to any other depth $z$ by applying Runge-Kutta to equation (30). However, for an accurate numerical extrapolation, the depth range of the extrapolation must be partitioned on a scale comfortably small with respect to the scale over which $\xi_{z}$ and $p$ characteristically vary. Elementary considerations with respect to the vertical energy flux of propagating waves generally render the amplitudes of $\xi_{z}$ and $p$ to be roughly in the following proportions:

$$
\left|\xi_{z}\right| \sim\left(c_{0} \rho_{0}\right)^{-1 / 2}
$$

and

$$
|p| \sim\left(c_{0} \rho_{0}\right)^{+1 / 2}
$$

The numerical extrapolation is therefore considerably more stable in equations for the vertical evolution of the variables

$$
\zeta \equiv\left(c_{0} \rho_{0}\right)^{1 / 2} \omega \xi_{z}
$$

and

$$
\eta \equiv\left(c_{0} \rho_{0}\right)^{-1 / 2} p
$$

The equation that evolves $(\zeta, \eta)$ retains the same basic form as equation (30):

$$
\omega^{2} \frac{\partial}{\partial z}\left(\begin{array}{l}
\zeta \\
\eta
\end{array}\right)=\boldsymbol{M}(z)\left(\begin{array}{l}
\zeta \\
\eta
\end{array}\right)
$$

with

$\boldsymbol{M}=\left[\begin{array}{cc}\left(\omega^{2} \beta-k^{2} g\right) & -\left(\omega^{2} / c_{0}^{2}-k^{2}\right)\left(\omega c_{0}\right) \\ \left(\omega^{4}-\omega^{2} \omega_{T}^{2}-k^{2} g^{2}\right) /\left(\omega c_{0}\right) & -\left(\omega^{2} \beta-k^{2} g\right)\end{array}\right]$,

where

$$
\beta \equiv \frac{1}{2} \frac{d}{d z}\left(\ln \rho_{0}+\ln c_{0}\right)
$$

is the scale depth of $\left(\rho_{0} c_{0}\right)^{1 / 2}$ and $\boldsymbol{M}$ otherwise contains no reference to rapidly evolving $\rho_{0}$.

To separate the purely upward- and downward-propagating modes at any particular depth, $z_{0}$, we consider the case in which $\boldsymbol{M}$ is constant,

$$
\boldsymbol{M}(z)=\boldsymbol{M}\left(z_{0}\right)
$$

in the neighborhood of $z_{0}$. In that case, equation (37) has analytic solutions of the form

$$
\left(\begin{array}{l}
\zeta \\
\eta
\end{array}\right)=\left(\begin{array}{c}
\zeta_{0} \\
\eta_{0}
\end{array}\right) e^{i \kappa z}
$$

with the relation between $\kappa, k$, and $\omega$ fixed by the requirement that the determinant of

$$
\begin{aligned}
& \boldsymbol{M}-\omega^{2} i \kappa \boldsymbol{I}= \\
& {\left[\begin{array}{cc}
\left(\omega^{2} \beta-k^{2} g\right)-\omega^{2} i \kappa & -\left(\omega^{2} / c_{0}^{2}-k^{2}\right)\left(\omega c_{0}\right) \\
\left(\omega^{4}-\omega^{2} \omega_{T}^{2}-k^{2} g^{2}\right) /\left(\omega c_{0}\right) & -\left(\omega^{2} \beta-k^{2} g\right)-\omega^{2} i \kappa
\end{array}\right]}
\end{aligned}
$$

must vanish. This leads to the secular equation,

$$
\begin{aligned}
\omega^{4}\left\{\omega^{2}\right. & \left.-\left[\omega_{T}^{2}+\left(\beta^{2}+\kappa^{2}\right) c_{0}^{2}\right]\right\} \\
& -\omega^{2}\left[\omega^{2}-\left(2 \beta g-g^{2} / c_{0}^{2}+\omega_{T}^{2}\right)\right] k^{2} c_{0}^{2}=0 .
\end{aligned}
$$

The secular equation is solved by $\omega=0$, indicating the existence of perturbations that remain static. For nonzero values of $\omega$, the secular equation is easily reduced to an equation for $\kappa$ in terms of $\omega$ and $k$ :

$$
\kappa= \pm \sqrt{\left(\frac{\omega^{2}-\omega_{a}^{2}}{c_{0}^{2}}\right)-\left(1-\frac{\omega_{g}^{2}}{\omega^{2}}\right) k^{2}}
$$

where the characteristic frequencies $\omega_{a}$ and $\omega_{g}$ are defined here by

$$
\omega_{a}^{2}=\beta^{2} c_{0}^{2}+\omega_{T}^{2},
$$

and

$$
\omega_{g}^{2}=2 \beta g-\frac{g^{2}}{c_{0}^{2}}+\omega_{T}^{2} .
$$

The characteristic frequency $\omega_{g}$ should not be confused with the familiar Brunt-Väisälä frequency, $N$, from which it differs by the relation

$$
\omega_{g}^{2}=N^{2}+g \frac{d}{d z} \ln c_{0}+\omega_{T}^{2}
$$

Deep in the convection zone, where $\omega_{a}$ and $\omega_{g}$ can be neglected, $\kappa$ reduces to

$$
\kappa= \pm \sqrt{\frac{\omega^{2}}{c_{0}^{2}}-k^{2}}
$$

which is the integrand of the eikonal integral expressed by equation (14). Where $\kappa$ is imaginary, acoustic waves are essentially excluded, or able to penetrate a limited depth as the incident wave flux is turned around and reflected back toward the solar surface. Where $\kappa$, is real, waves can be roughly regarded as freely propagating. The purely downward- or upward-propagating modes are represented by the eigenvectors, $\left(\zeta_{ \pm}, \eta_{ \pm}\right)$, of $\boldsymbol{M}$ corresponding to the respective positive or negative values of $i \kappa$ to satisfy the secular equation (44). These conveniently allow us to select the wave mode imposed by whichever of Assumptions 1 and 2 apply.

In general, an amplitude $(\zeta, \eta)$ that is an eigenvector of $\boldsymbol{M}$ at one depth will not be an eigenvector at another depth where $\boldsymbol{M}$ is significantly different. However, throughout a depth range over which

$$
\left|\frac{d \kappa}{d z}\right| \ll \kappa^{2}
$$




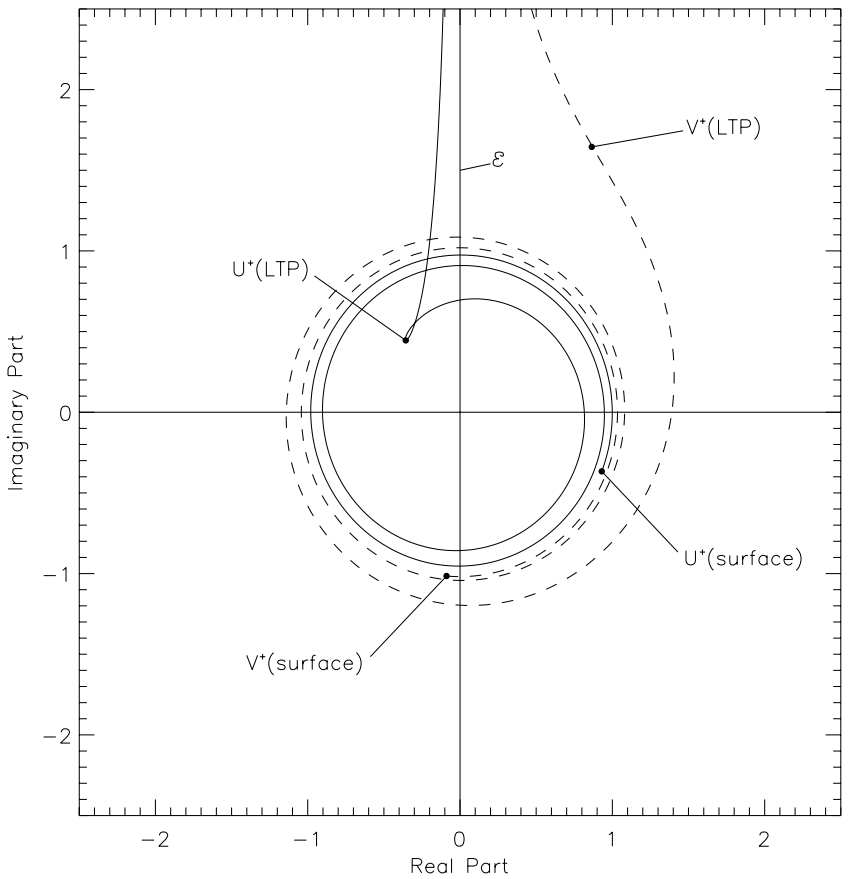

FIG. 2.-Complex amplitudes $U^{+}$(solid curve) and $V^{+}$(dashed curve) are plotted for horizontal spatial frequency $l=k R_{\odot}=300$ and temporal frequency $\nu=\omega / 2 \pi=5 \mathrm{mHz}$. The loci of $U^{+}$and $V^{+}$wind counterclockwise (increasing phase) about the origin as depth $z$ increases from the surface. As $z$ passes the lower turning point (LTP) $U^{+}$and $V^{+}$explode along the positive imaginary axis.

the progression of $(\zeta, \eta)$ can be regarded as "adiabatic," in the sense that if it began as an eigenvector, it will evolve so as to adhere closely to that eigenvector throughout such a range. When $\kappa$ changes suddenly, equation (49) fails, and specular reflections are generally created, contaminating either single mode with the one propagating in the opposite direction on at least one side or the other of the anomaly. Familiar examples of reflections caused by the failure of equation (49) are those characterizing the surface of a pond or window glass.

The condition stated by equation (49) must eventually fail altogether for substantial values of $k$ at the lower turning point, $z_{l}$, of the wave, at which the sound speed, $c_{0}$, becomes sufficient to drive $\kappa^{2}$ negative. When $\kappa^{2}$ becomes negative, $i \kappa$ becomes real and the solutions of equation (37) explode or collapse, as suggested by equation (41). A mode that is purely upward- or downward-propagating where equation (49) is satisfied invariably contains an exploding component when $\kappa^{2}$ becomes negative. Where $\kappa^{2}$ is positive, $\zeta_{+}$and $\eta_{+}$wind counterclockwise in the complex plane around the origin (a downward-propagating wave) as shown in Figure 2. As $\kappa^{2}$ plummets through zero, the loci of $\zeta_{+}$and $\eta_{+}$straighten out and accelerate rapidly outward, converging toward an asymptote, $\mathcal{E}$, whose argument depends on the initial argument assigned to $\zeta_{+}$. The lower turning point is indicated in Figure 2 on the loci of $\zeta_{+}$and $\eta_{+}$by the label "LTP." In this example we have chosen the initial argument of $\zeta_{+}$so that the asymptote, $\mathcal{E}$, lies along the positive imaginary axis. In that case, the loci of $\zeta_{+}$ and $\eta_{+}$make a figure resembling a backward G-clef centered at the origin. In this perspective we represent $\zeta_{ \pm}$and $\eta_{ \pm}$by the elementary complex functions $U^{ \pm}$and $V^{ \pm}$, respectively, with real and imaginary decompositions defined as follows:

$$
\begin{aligned}
U^{ \pm}(k, \omega, z) & =U_{r}(k, \omega, z) \pm i U_{i}(k, \omega, z), \\
V^{ \pm}(k, \omega, z) & =V_{r}(k, \omega, z) \pm i V_{i}(k, \omega, z) .
\end{aligned}
$$
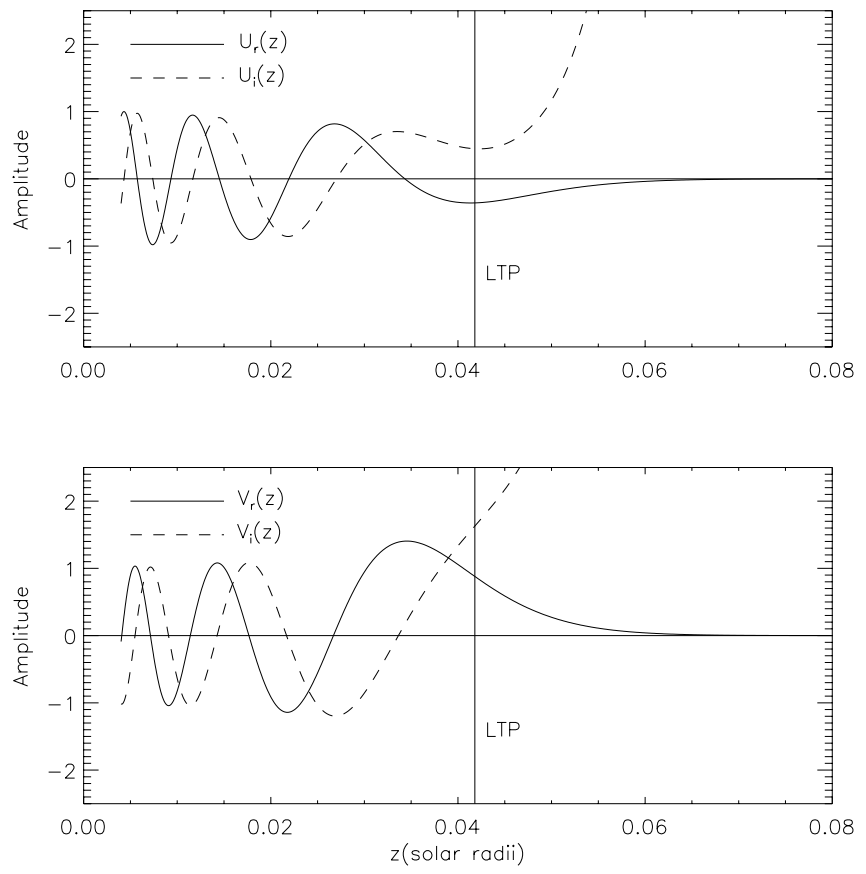

FIG. 3.- Real and imaginary parts of amplitudes $U^{+}$(top, normalized vertical displacement) and $V^{+}$(bottom, normalized pressure) are plotted as a function of depth $z$ for horizontal spatial frequency $l=300$ and temporal frequency $\nu=\omega / 2 \pi=5 \mathrm{mHz}$.

In general, $\left(U^{+}, V^{+}\right)$represents a purely downward-propagating wave, while $\left(U^{-}, V^{-}\right)$represents an upward-propagating one. The elementary real and imaginary components, $U_{r}, U_{i}, V_{r}$, and $V_{i}$ are plotted in Figure 3 as functions of depth. Because $U_{i}$ and $V_{i}$ explode violently beneath the lower turning point, we infer that they cannot be substantial constituents in acoustic disturbances initiated anywhere near the solar surface. It is nevertheless useful for a broad variety of diagnostic purposes to express acoustic disturbances in the shallow subphotosphere in terms of $\left(U^{+}, V^{+}\right)$and $\left(U^{-}, V^{-}\right)$, which contain both of these components. We will address these considerations in the following section.

The modes $U^{ \pm}$normalized to unity at the surface serve as the basic element of the Green's functions, $\hat{G}_{\mp}$, for the superjacent vantage in the standard formalism of computational seismic holography in the Fourier domain, as represented by equation (5), where $\hat{\psi}(\boldsymbol{k}, \omega)$ is equated to the vertical displacement, $\hat{\xi}_{z}$, of the solar medium at the surface. Its associates, $V^{ \pm}$, serve the same function for the regression of the pressure, given observations of only the vertical displacement. Figure 4 compares the phases of $U^{-}$and $V^{-}$with the eikonal, $\tilde{S}$, prescribed by equation (14).

For an active region near disk center, the MDI observations give us a close approximation to the vertical velocity, $\dot{\zeta}=\partial \zeta / \partial t$. Acoustic progressions applied to the MDI observations literally as prescribed by the formalism following equations (19), (20), and (21) therefore render representations of the time derivative, $\dot{\zeta}$, of the normalized vertical displacement, $\zeta$. Under Assumption 1 or 2 the formalism likewise gives a Green's function for $\eta$ based on the surface value if $\zeta$. This offers us the alternative of a focal-plane representation of the time derivative, $\dot{\eta}$, of the normalized acoustic pressure perturbation from the MDI observations on similar terms. 


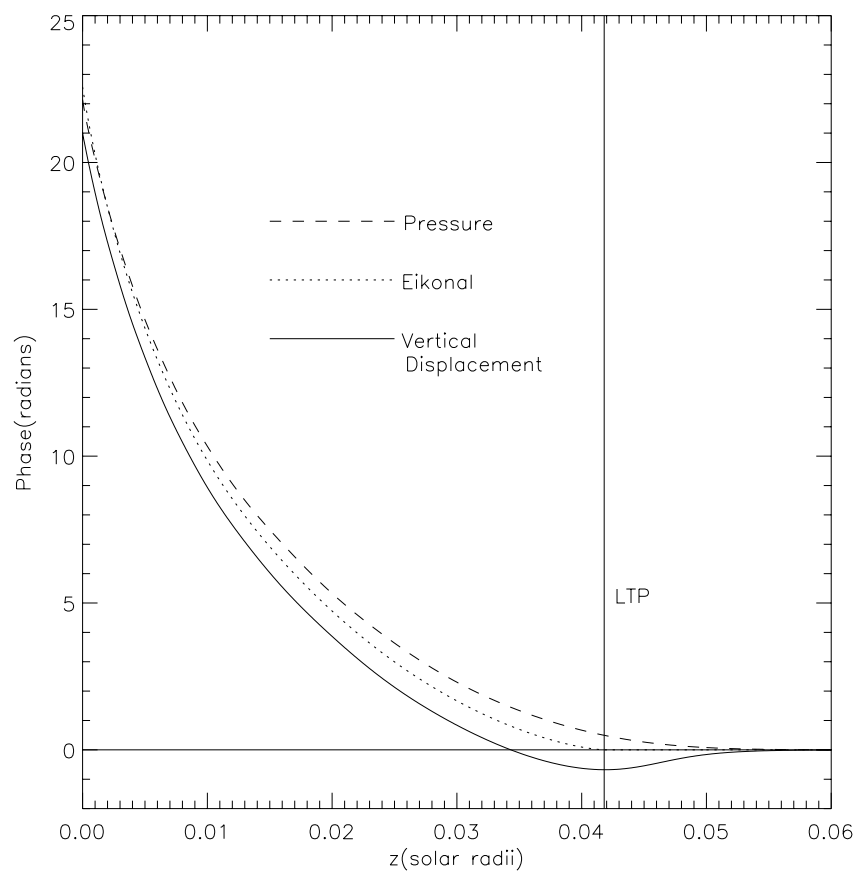

FIG. 4.-Phases of amplitudes $U^{-}$(solid curve) and $V^{-}$(long-dashed curve) are compared with the eikonal, $\tilde{S}$ (short-dashed curve), for horizontal spatial frequency $l=300$ and temporal frequency $\nu=\omega / 2 \pi=5 \mathrm{mHz}$. The latter is integrated from the lower turning point (LTP).

\subsection{Reflections and Vantages}

General acoustic disturbances in the subphotosphere for any frequency, $\omega$, can be expressed in terms of superpositions of the amplitudes $\left(U^{ \pm}, V^{ \pm}\right) \equiv\left(U_{r} \pm i U_{i}, V_{r} \pm i V_{i}\right)$. However, as mentioned before, real physical disturbances can only be characterized by superpositions for which the coefficients of the exploding components, $i\left(U_{i}, V_{i}\right)$, are null. The exploding components represent waves that would have to be driven by a source beneath the lower turning point. The physical interpretation is that a wave $\left(U_{r}+i U_{i}, V_{r}+i V_{i}\right)$ created by a source at the solar surface is reflected at the lower turning point. The resulting upcoming wave, $\left(U_{r}-i U_{i}, V_{r}-i V_{i}\right)$, superposed onto the downward-propagating wave reinforces the $\left(U_{r}, V_{r}\right)$ component and annihilates the $i\left(U_{i}, V_{i}\right)$ contribution by destructive interference. As one sees from the plots that appear in Figure 3, the standing wave, represented by $\left(U_{r}, V_{r}\right)$, penetrates a limited distance beneath the lower turning point, its amplitude collapsing as rapidly as the amplitude of $\left(U_{i}, V_{i}\right)$ explodes.

In fact, it is useful for various diagnostic purposes to have the facility to discriminate between upward- and downwardpropagating components, even while neither is thought to be present without the other in the real solar subphotosphere. The analyst can decide whether to include either component without the other or include both components in his representation of the Green's function, $G_{-}$. This discrimination renders a basis in the Fourier domain for the distinction between superjacent and subjacent vantage holography (see Fig. 1).

For frequencies $\nu=\omega /(2 \pi)$ less than $\sim 5 \mathrm{mHz}$, an upper turning point occurs in the neighborhood of the solar photosphere, when $\omega_{a}$ exceeds $\omega$ and drives $\kappa^{2}$ negative for all values of $k$. This makes the solar subphotosphere an efficient reflector of waves whose frequencies are less than $\sim 5 \mathrm{mHz}$. As with reflections from the lower turning point, the analyst has the option of representing the surface reflections in the Green's function, and this is the basis of "multiple-skip holography," illustrated in Figures 6 and 7 of Braun \& Lindsey (2000a).

Waves with frequencies $\nu$ above $\sim 5 \mathrm{mHz}$ ascend substantially into the photosphere before a turning point is encountered. At this point it is apparent that the adiabatic representation conveyed by equation (16) is no longer entirely satisfactory. A major dynamic factor for which equation (16) does not account is strong acoustic damping as a result of the escape of electromagnetic radiation directly into space. It is empirically clear that acoustic radiation emerging to the quiet solar surface from below leaves an observable surface signature on its arrival. However, there is little evidence of a coherent specular reflection at the higher frequencies. Consequently, multiple-skip holography of the quiet Sun fails at frequencies above about $5 \mathrm{mHz}$. As in electromagnetic optics, it is these high frequencies that offer the greatest spatial discrimination in wave diagnostics.

\subsection{The Local Control Correlations}

An important control experiment for holographic projections is accomplished by drawing the focus to the solar surface, as illustrated in Figure 1b, and comparing ingressions and egressions separately with the acoustic amplitude, $\psi$, observed at the focus (see $\S 8.2$ of Lindsey \& Braun 2000b). The correlation

$$
C_{\mathrm{LC}-}(\boldsymbol{r}, 0) \equiv\left\langle\psi(\boldsymbol{r}, \omega) H_{-}^{*}(\boldsymbol{r}, 0, \omega)\right\rangle_{\Delta \omega}
$$

compares the local acoustic amplitude with waves focused into the surface focal point, $\boldsymbol{R}=(\boldsymbol{r}, 0)$, from the surrounding pupil. The time-reverse correlation,

$$
C_{\mathrm{LC}+}(\boldsymbol{r}, 0) \equiv\left\langle H_{+}(\boldsymbol{r}, 0, \omega) \psi^{*}(\boldsymbol{r}, \omega)\right\rangle_{\Delta \omega},
$$

tells us how a local surface disturbance at $(\boldsymbol{r}, 0)$ propagates outward to resurface in the pupil minutes later. We apply the holographic progressions representing vertical displacement to MDI line-of-sight Doppler observations of a region of quiet Sun centered at solar disk center, equating $\psi$ to the normalized vertical velocity, $\dot{\zeta}$, at the base of the photosphere. The holographic projections applied to the MDI observations then likewise render a representation of $\dot{\zeta}$, as explained at the end of $\S 5.1$. We will call $C_{\mathrm{LC}-}$ and $C_{\mathrm{LC}+}$ the "local control correlations," or just the "control correlations" for short.

Figure 5 shows comparative statistics of the normalized control correlations,

$$
\hat{C}_{\mathrm{LC} \pm} \equiv \frac{C_{\mathrm{LC} \pm}}{\sqrt{\left\langle\left|H_{ \pm}\right|^{2}\right\rangle\left\langle|\psi|^{2}\right\rangle}},
$$
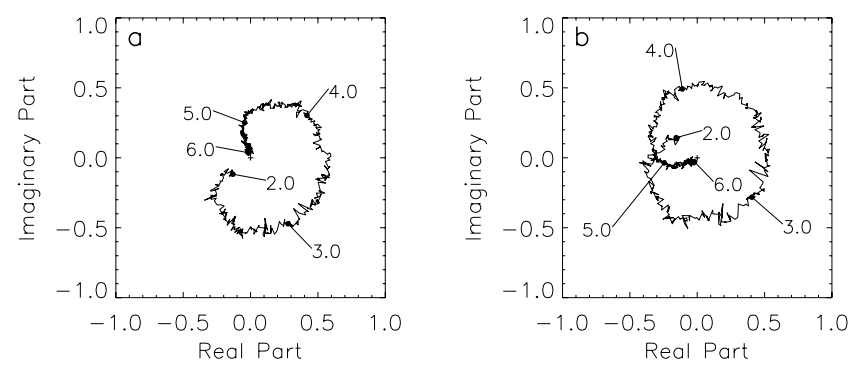

FIG. 5.-Normalized control correlation $\hat{C}_{\mathrm{LC}-}$ (see eq. [52]) for $(a)$ the quiet-Sun hydromechanical and $(b)$ the eikonal extrapolations are plotted over the $2-7 \mathrm{mHz}$ spectrum in the left and right panels, respectively, with $\psi$ in eqs. (51) and (52) equated to the normalized vertical velocity, $\dot{\zeta}$. Frequencies are labeled in $\mathrm{mHz}$ along the respective loci. 

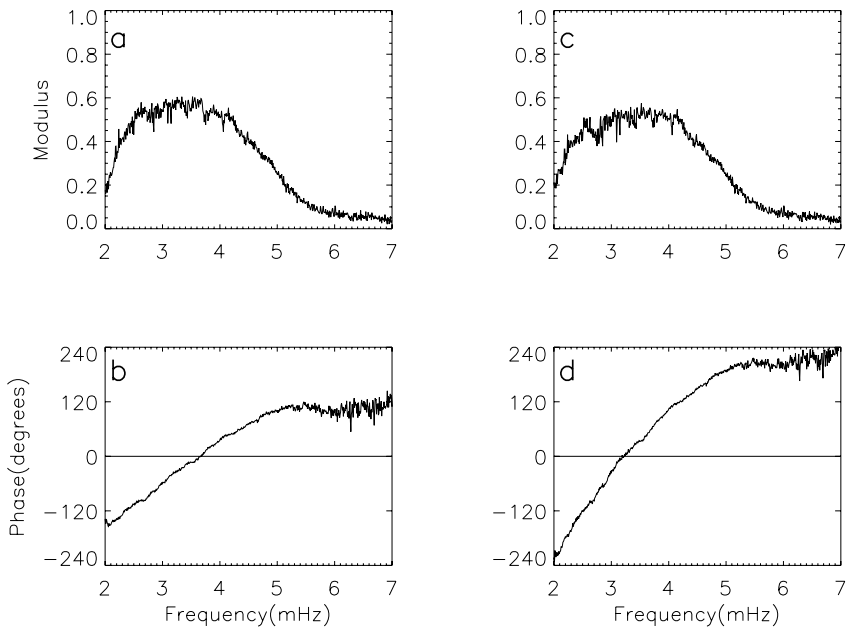

FIG. 6.-Moduli and phases of the quiet-Sun control correlation, $\hat{C}_{\mathrm{LC}-}$, plotted in Fig. 5, are plotted here as functions of frequency. Left column shows (a) modulus and $(b)$ phase of the hydromechanical correlation in which $\psi$ is the vertical velocity. Right column shows $(c)$ modulus and $(d)$ phase under the eikonal extrapolation.

for the quiet Sun, computed hydromechanically for $\dot{\zeta}$ (Fig. 5a) and using the eikonal approximation (Fig. $5 b$ ). In each case the acoustic progressions were computed over an annular pupil 15-45 $\mathrm{Mm}$ in radial extent. These are plotted in the complex plane over the range $2-7 \mathrm{mHz}$. Frequencies are labeled in $\mathrm{mHz}$ along the respective loci. It is the spatial averages of $\hat{C}_{\mathrm{LC}+}$ and $\hat{C}_{\mathrm{LC}-}$ over a substantial area that are actually plotted in Figure 5. The two are indistinguishable in the quiet Sun, but become unmistakably different when the focus is in an active region photosphere. Figure 6 shows the amplitudes and phase of the normalized correlations plotted in Figure 5 as functions of frequency.

The difference between the hydromechanical and eikonal phases profiles (Figs. $6 b$ and $6 d$ ) renders an appraisal of the effects of dispersion that escape the eikonal computation. This partially addresses issues raised by Barnes \& Cally (2001) regarding the inability of the eikonal computation by itself to account for dispersion. It is nevertheless evident that even the hydromechanical phase varies substantially over the $2-7 \mathrm{mHz}$ range.

\subsection{The Phase Progression}

We will proceed here to address considerations that bear on the hydromechanical phase progression in the control correlations. The theoretical framework needed for a realistic interpretation of the correlations plotted in Figures 5 and 6 has not been worked out in its entirety. This includes considerations such as how upcoming waves are reflected back into the solar interior and the effects of electromagnetic radiative damping, for which equation (16) contains no account. It is apparent that upcoming waves above about $5 \mathrm{mHz}$ are strongly absorbed by the overlying photosphere or chromosphere (Duvall et al. 1993; Lindsey \& Braun 1999; Braun \& Lindsey 2000b). It is actually possible that the upcoming waves are substantially reflected but with scrambled phases. In any case, they are somehow replaced by down-going waves that have no apparent phase relation with the originals. The high-frequency waves are of special interest because of the relatively fine spatial discrimination they offer. The correlations plotted in Figures 5 and 6 are probably diagnostic of important aspects of how the waves are generated,
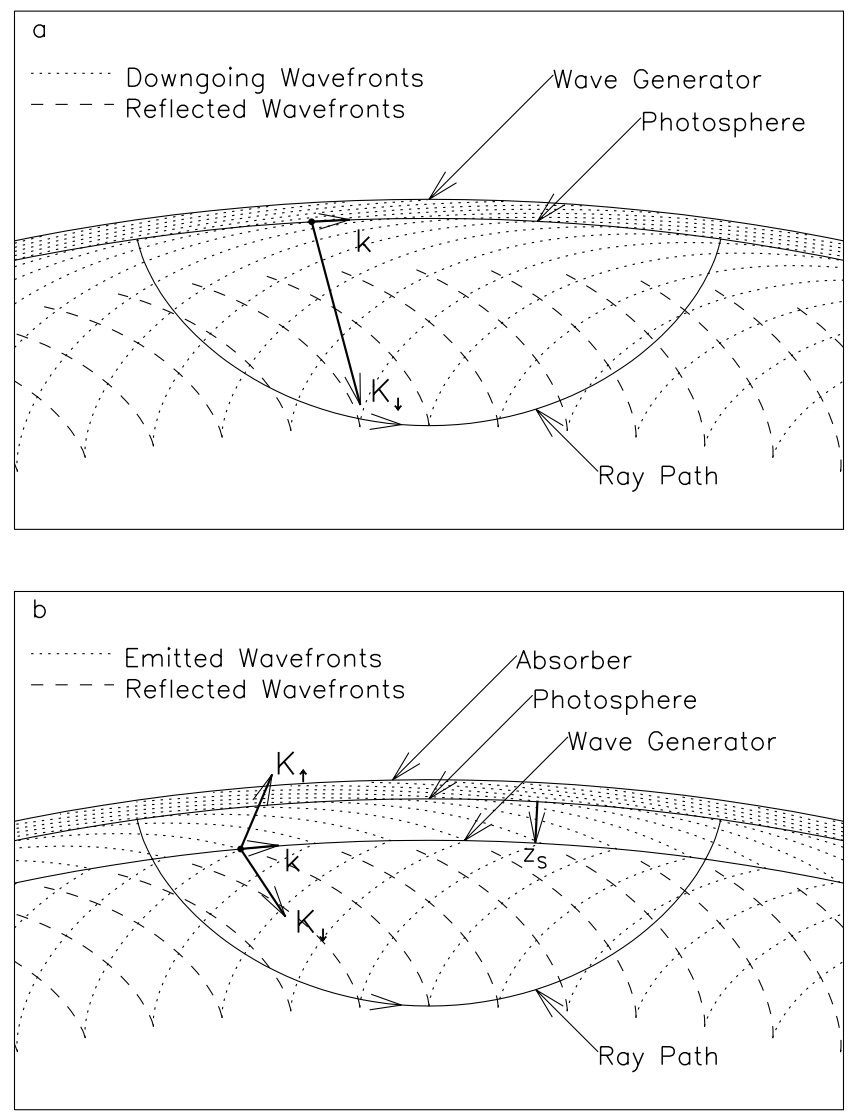

FIG. 7.-Diagrams showing wave fronts for downward-propagating (shortdashed curves) and upward-propagating (long-dashed curves) components of a wave mode driven at $(a)$ a surface above the photosphere and $(b)$ by a surface at depth $z_{s}$ beneath the photosphere. Arrows labeled $\boldsymbol{K}_{\uparrow \downarrow}$ indicate gradients of the phase for the upward- and downward-propagating components at the photosphere in $(a)$ and the driving surface in $(b)$. The arrows labeled $\boldsymbol{k}$ indicate the horizontal component of $\boldsymbol{K}_{\uparrow \downarrow}$. Solid curves indicate the ray path of least travel time.

particularly at the higher frequencies, where specular reflections from the photosphere are relatively weak.

We will not attempt a realistic analysis of any of the factors that give rise to the phase progression in this paper. However, we will suggest some elements of formalism that could facilitate such an examination. To clarify some of the important distinctions, we begin with a control model under which the phase progression of the local control correlations is null. We will call this reference model, which is illustrated in Figure $7 a$, "Acoustic Model 0." Model 0 assumes that the waves we see at the solar surface are emitted downward from an overlying surface, above the base of the photosphere where we observe the disturbances that manifest them. In such a model, all waves register in helioseismic observations twice: The first registration, represented by the field $\psi_{\downarrow}$, is that of the wave passing downward through the base of the photosphere on its way into the solar interior. The second, $\psi_{\uparrow}$, is that of the upward-propagating echo on its way back to the source layer. The helioseismic observations generally register the sum,

$$
\psi=\psi_{\downarrow}+\psi_{\uparrow}
$$

and are unable to distinguish between the individual components, $\psi_{\downarrow}$ and $\psi_{\uparrow}$. Consequently, when we compute an acoustic 
progression, as prescribed by equation (4), we are computing the sum,

$$
H_{ \pm}=H_{\uparrow \pm}+H_{\downarrow \pm},
$$

where

$H_{\uparrow \downarrow \pm}(\boldsymbol{r}, z, \omega) \equiv \int_{\mathcal{P}} d^{2} \boldsymbol{r}^{\prime} G_{ \pm}\left(\left|\boldsymbol{r}-\boldsymbol{r}^{\prime}\right|, z, \omega\right) \psi_{\uparrow \downarrow}\left(\boldsymbol{r}^{\prime}, \omega\right)$.

This is somewhat unfortunate, since we are only actually interested in $H_{\downarrow-}$ and in $H_{\uparrow+}$. When computing the control correlations, $\left\langle\psi H_{-}^{*}\right\rangle$ and $\left\langle H_{+} \psi^{*}\right\rangle$, the undesired acoustic progressions $H_{\uparrow-}$ and $H_{\downarrow+}$ contribute noise.

The correlation of diagnostic interest contained in $C_{\mathrm{LC}+}$, for example, can be expressed by the component

$$
C_{\mathrm{LC} \uparrow+}(\boldsymbol{r}, 0) \equiv\left\langle H_{\uparrow+}(\boldsymbol{r}, 0, \omega) \psi_{\downarrow}^{*}(\boldsymbol{r}, \omega)\right\rangle_{\Delta \omega},
$$

representing the egression, $H_{\uparrow+}(\boldsymbol{r}, 0, \omega)$, of just the upcoming radiation in the pupil correlated with only the radiation emitted downward from the focal point. Because we cannot distinguish the up-going from the down-going components, the full correlation, $C_{\mathrm{LC}+}$, contains the foregoing component, $C_{\mathrm{LC} \uparrow \downarrow+}$, plus three "ghost" components,

$$
C_{\mathrm{LC}+}=C_{\mathrm{LC} \uparrow \downarrow+}+C_{\mathrm{LC} \uparrow \uparrow+}+C_{\mathrm{LC} \downarrow \downarrow+}+C_{\mathrm{LC} \downarrow \uparrow+},
$$

where we generalize the correlation $C_{\mathrm{LC} \uparrow \downarrow+}$, defined by equation (57), by the formalism

$$
C_{\mathrm{LC} \mu \nu \pm}(\boldsymbol{r}, 0) \equiv\left\langle H_{\mu \pm}(\boldsymbol{r}, 0, \omega) \psi_{\nu}^{*}(\boldsymbol{r}, 0, \omega)\right\rangle_{\Delta \omega},
$$

with $\mu, \nu \in\{\uparrow, \downarrow\}$.

In the case of the local correlations, the expectations of the ghosts $C_{\mathrm{LC} \uparrow \uparrow \pm}$ and $C_{\mathrm{LC} \downarrow \downarrow \pm}$ turn out to be essentially null, and $C_{\mathrm{LC} \downarrow \uparrow+}$ and $C_{\mathrm{LC} \uparrow \downarrow-}$ are nearly so for large pupils, such as for an annulus whose radial range corresponds to a range of time delays that is many times the period of the wave. The ghosts then simply contribute neutral noise to $C_{\mathrm{LC} \pm}$ with a nearly null expectation. If Model 0 were accurate, both $C_{\mathrm{LC}+}$ and $C_{\mathrm{LC}-}$ would be real and positive, provided that the acoustic progressions were computed properly.

The phase progression shown by Figures 5 and 6 reflect attributes of the real solar photosphere that Model 0 does not possess. This includes strong specular reflections from the upper subphotosphere and photosphere in the $2-5 \mathrm{mHz}$ spectrum and radiative damping, not included in equation (16). It is also evident that the $p$-modes are generated beneath the photosphere (Kumar 1994; Kumar \& Basu 2000), not above it. In the $2-5 \mathrm{mHz}$ spectrum the solar photosphere reflections are certain to be the predominant factor in the phase progression of the control correlations. Above $5 \mathrm{mHz}$ the reflectivity appears to diminish rapidly with frequency, suggesting a model for high frequencies that retains the absorber at the upper boundary of Model 0.

We will proceed accordingly to consider the acoustic model illustrated by Figure $7 b$, which we will call "Acoustic Model 1." In this model waves are emitted both upward and downward from a surface submerged to depth $z_{S}$. For simplicity we suppose that all waves passing upward through the photosphere continue to be absorbed by an overlying surface, as illustrated.

The formalism needed to represent Model 1 is different from that for Model 0 in a number of important respects. The down-going field, $\psi_{\downarrow}$, of its predecessor no longer exists. What appears in its place is a field propagating upward from the source layer. In the following discussion, we will use the field propagating upward from the source layer as a proxy for the wave propagating downward from it at the same angle. We will now represent the relevant amplitudes in the spatially Fourier transformed domain. The amplitudes of the upwardand downward-propagating waves emanating from the source layer will be represented by $\hat{S}_{\uparrow}$ and $\hat{S}_{\downarrow}$, respectively. For a downward-propagating wave of amplitude $\hat{S}_{\downarrow}$ with a local wavenumber $\boldsymbol{K}_{\downarrow}=(\boldsymbol{k}, \kappa)$ and frequency $\omega$, there is an upwardpropagating wave, $\hat{S}_{\uparrow}$, with wavenumber $\boldsymbol{K}_{\uparrow}=(\boldsymbol{k},-\kappa)$ at that same frequency. Because we propose to use the surface disturbance caused by the upward-propagating component as a proxy for the downward-propagating wave, we represent it by the symbol $\psi_{\downarrow}$, notwithstanding that it is now propagating upward, like the echo, $\psi_{\uparrow}$, of its initially downward-propagating counterpart. The use of $\psi_{\downarrow}$ for such a proxy clearly depends on a significant statistical correlation, $\left\langle\hat{S}_{\downarrow} \hat{S}_{\uparrow}^{*}\right\rangle$, between $\hat{S}_{\downarrow}$ and $\hat{S}_{\uparrow}$.

The acoustic progression from depth $z_{S}$ to the surface is represented by applying the appropriate Green's function to $\hat{S}_{\uparrow}$ :

$$
\hat{\psi}_{\downarrow}=\hat{G}_{\uparrow-} \hat{S}_{\uparrow}
$$

Here $\hat{G}_{\uparrow-}$ represents both the propagation of the wave from source to surface and any additional surface dynamics that influence how the wave actually registers as a helioseismic signature, $\hat{\psi}_{\downarrow}$.

The downward-propagating wave, $\hat{S}_{\downarrow}$, proceeds into the solar interior, penetrating to the lower turning point, $z_{l}$, before it is refracted back to the surface to register the signature $\hat{\psi}_{\uparrow}$. Let $\hat{G}_{\downarrow-}$ represent the propagation of $\hat{S}_{\downarrow}$ from depth $z_{S}$ to $z_{l}$ and back up to depth $z_{S}$. We then apply $\hat{G}_{\uparrow-}$ to $\hat{G}_{\downarrow} \hat{S}_{\downarrow}$ to complete the propagation of the reflected wave the remaining vertical distance, $z_{S}$, back to the surface:

$$
\hat{\psi}_{\uparrow}=\hat{G}_{\uparrow-} \hat{G}_{\downarrow-} \hat{S}_{\downarrow} .
$$

For the following discussion we will distinguish between the Green's functions, $G_{ \pm}^{C}$, that characterize the acoustic model for purposes of computing holographic progressions, $H_{ \pm}$, and those discussed above, $\hat{G}_{ \pm}$, which we will suppose characterize the real solar atmosphere so as to include details of which we may not be entirely aware. The holographic components of interest in phase correlation diagnostics are

$$
\begin{aligned}
& \hat{H}_{\downarrow-}=\hat{G}_{\downarrow-}^{C} \hat{\psi}_{\downarrow}, \\
& \hat{H}_{\uparrow+}=\hat{G}_{\downarrow+}^{C} \hat{\psi}_{\uparrow} .
\end{aligned}
$$

Applying equations (60) and (61) to equation (62) as appropriate, it follows directly that the correlations representing the substantial statistical components of $C_{\mathrm{LC}+}$ (see eqs. [57] and [58]) and $C_{\mathrm{LC}-}$ are related to $S_{\uparrow}$ and $S_{\downarrow}$ by

$$
\left\langle\hat{H}_{\uparrow+} \hat{\psi}_{\downarrow}^{*}\right\rangle=\left\langle\hat{\psi}_{\uparrow} \hat{H}_{\uparrow-}^{*}\right\rangle=\left|\hat{G}_{\uparrow-}\right|^{2}\left(\hat{G}_{\downarrow+}^{C} \hat{G}_{\downarrow-}\right)\left\langle\hat{S}_{\downarrow} \hat{S}_{\uparrow}^{*}\right\rangle .
$$

The correlation, $\left\langle\hat{S}_{\downarrow} \hat{S}_{\uparrow}^{*}\right\rangle$, between $\hat{S}_{\downarrow}$ and $\hat{S}_{\uparrow}$ is certain to be quite strong under certain conditions, for example if the waves are generated exclusively by dipole emitters in a thin layer. However, if wave generation is characterized by independently phased sources distributed over a half-wavelength or more in depth, then $\left\langle\hat{S}_{\downarrow} \hat{S}_{\uparrow}^{*}\right\rangle$ can be considerably reduced. 
For an intuitive discussion of the relationship expressed by equation (63), the downward-propagating disturbance can be regarded as having a head start with respect to phase travel time, by a margin

$$
\Delta t=\frac{1}{\omega} \int_{0}^{z_{S}} \kappa d z
$$

while the arrival of its upward-propagating counterpart at the surface, as the downward-propagating proxy, is delayed by $\Delta t$. While we should expect to see a strong correlation between the egression and the local amplitude based on the return of the wave to the solar surface some distance away, the phase delay between the local signature and the egression signature is reduced by $2 \Delta t$, giving rise to a phase progression of $-2 \omega \Delta t$ with frequency.

Below $\sim 5 \mathrm{mHz}$, the correlation amplitudes are dominated by strong specular reflections of upcoming waves back into the solar interior from various levels in or below the photosphere that depend on frequency. A detailed account of the reflections, not attempted here, will entail further phase and modulus corrections. Further phase shifts not accounted for in this study include those introduced in the shallow subphotosphere by radiative damping and by any errors in the acoustic model.

The small modulus of the normalized correlation in Figures $6 a$ and $6 c$ above $5 \mathrm{mHz}$ suggests a relatively low correlation, $\left\langle\hat{S}_{\downarrow} \hat{S}_{\uparrow}^{*}\right\rangle$, between the up-going and down-going radiation. This suggests that a predominant fraction of the highfrequency acoustic power originates from sources distributed over a range of order 1.0 Mm in depth, somewhat greater than that suggested by earlier work (Kumar 1994; Kumar \& Basu 2000). This and alternative possibilities need to be carefully examined.

In some applications, it is useful to correct the correlation phase shifts plotted in Figures 5 and 6 without consideration as to their interpretation in terms of acoustics. Phase-correlation imaging of active regions on the Sun's far surface, for example, depends on correlation statistics integrated over a fairly broad spectrum, of acoustic radiation that has been reflected from the surface several times. If the phase progression is not corrected, then contributions to the correlation signature from different frequencies will be subject to destructive interference, so that the full statistical value of the correlation over a substantial range, $\Delta \omega$, can be substantially lost. It can therefore be very profitable, as a general diagnostic utility, for the analyst to have the facility at his disposal for correcting these phase shifts before the correlation statistics are integrated over frequency. This is conveniently accomplished by fitting the phase curves plotted in Figure 6 to analytic functions and phase shifting the Green's functions accordingly before computing the acoustic progressions.

\subsection{Phase-Correlation Holography from the Lateral Vantage}

We now turn to the application of phase-correlation holography to the shallow subphotosphere. In the discussion that follows we will apply to seismic holography in the lateral vantage, whose generality is illustrated in Figures $1 c$ and $1 d$. In this configuration we compare the ingression, $H_{-}^{\mathcal{R}}(\boldsymbol{r}, z, \omega)$, over a pupil, $\mathcal{R}$, with the egression, $H_{+}^{\mathcal{L}}(\boldsymbol{r}, z, \omega)$, over a pupil $\mathcal{L}$ with a common focal point, $(\boldsymbol{r}, z)$, such that acoustic radiation passing from $\mathcal{L}$ to $\mathcal{R}$ through the neighborhood of $\boldsymbol{r}$ passes $\boldsymbol{r}$ laterally, on the average:

$$
C^{\mathcal{L} \mathcal{R}}(\boldsymbol{r}, z, \omega)=\left\langle H_{+}^{\mathcal{L}}(\boldsymbol{r}, z, \omega) H_{-}^{\mathcal{R} *}(\boldsymbol{r}, z, \omega)\right\rangle .
$$

In general, $\mathcal{L}$ and $\mathcal{R}$ may or may not be separate regions. Appropriately differentiated pupils can allow the analyst to discriminate between acoustic anomalies in the neighborhood of the focal point that are refractive in nature on one hand and advective along the axis of the cone of illumination of $\boldsymbol{r}$ on the other. The symmetric phase shift,

$$
\phi^{S} \equiv \frac{1}{2}\left(\arg C^{\mathcal{L} \mathcal{R}}+\arg C^{\mathcal{R L}}\right)
$$

is sensitive in the first order to refractive perturbations that retard or advance the phase of the radiation that passes through them equally in both directions. The antisymmetric phase shift,

$$
\phi^{A} \equiv \frac{1}{2}\left(\arg C^{\mathcal{L} \mathcal{R}}-\arg C^{\mathcal{R} \mathcal{L}}\right)
$$

is sensitive in the first order to Doppler perturbations, which advance the phase in the direction of motion and retard it against. Both $\phi^{S}$ and $\phi^{A}$ have higher order sensitivities under certain circumstances.

\subsection{Path Conjugality}

An important concept regarding the relationship between the ingression and egression pupils, $\mathcal{L}$ and $\mathcal{R}$, is that of mutual conjugality with respect to ray paths that connect the focus, $\boldsymbol{r}$, to the pupils. Let $\boldsymbol{r}^{\prime}$ be a point in $\mathcal{L}$, and $\Gamma_{\boldsymbol{r}, \boldsymbol{r}^{\prime}}$ be the ray path that connects $\boldsymbol{r}^{\prime}$ to the focal point, $\boldsymbol{r}$. Let $\boldsymbol{r}^{\prime \prime}$ be the point at which the continuation of $\Gamma_{\boldsymbol{r}, \boldsymbol{r}^{\prime}}$ past the focal point returns to the surface. We will call $\boldsymbol{r}^{\prime \prime}$ the conjugate point of $\boldsymbol{r}^{\prime}$. We will call the set, $\mathcal{P}^{\prime}$, of all points that are conjugate to the points in a pupil $\mathcal{P}$ for focus $\boldsymbol{r}$ the conjugate pupil of $\mathcal{P}$ with respect to $\boldsymbol{r}$, and say that $\mathcal{P}$ and $\mathcal{P}^{\prime}$ are mutually conjugate (with respect to $\boldsymbol{r}$ ). When the ingression and egression pupils, $\mathcal{R}$ and $\mathcal{L}$ are mutually conjugate, as they are in Figures $1 c$ and $1 d$, the correlations $C^{\mathcal{L} \mathcal{R}}$ and $C^{\mathcal{L R}}$ will generally be quite strong in the absence of acoustic anomalies. In that case, the function of a local anomaly in the neighborhood of the focal point when $\mathcal{L}$ and $\mathcal{R}$ are mutually conjugate is to phase shift or otherwise perturb a correlation that is nominally strong. The analyst might alternatively fashion $\mathcal{L}$ and $\mathcal{R}$ to avoid each other's conjugates, whereby $C^{\mathcal{L} R}$ and $C^{\mathcal{L R}}$ would devolve to nominally null values that would be disturbed by a strong compact scatterer. According to our understanding a relatively extended but subtle anomaly that only slightly deflects acoustic radiation passing through it will generally render a more definite signature, in terms of a phase shift, when the pupils are devised to maximize the correlation in the absence of such an anomaly.

\subsection{Phase-Correlation Holography of the Quiet Subphotosphere}

In the discussion that follows we will concentrate our attention on refractive anomalies and their effect on $\phi^{S}$ when $C^{\mathcal{L} \mathcal{R}}$ and $C^{\mathcal{R} \mathcal{L}}$ are optimized in the absence of anomalies. The pupil, $\mathcal{P}$, for both ingression and egression computations will be the complete self-conjugate annulus, encompassing the endpoints of all ray paths that pass through the focal point at angles up to $\pm 45^{\circ}$ from the horizontal plane thereat. As illustrated by Figure $1 d$, the pupil shrinks as the focal point approaches the surface. For zero depth the inner radius of the pupil shrinks to zero, while the outer radius approaches a welldefined limit, the surface-to-surface skip distance of a ray whose tangent angle is $45^{\circ}$ at the surface path angle from the 
horizontal plane at the surface focal point. In the case of the full-disk MDI observations, the spectrum of the $5 \mathrm{mHz}$ Green's function expands to include wavenumbers that approach the resolution limit of the full-disk MDI images for focal depths substantially less than $4 \mathrm{Mm}$.

Figure 8 shows statistics of the "normalized ingressionegression correlation,"

$$
\hat{C} \equiv \frac{C}{\sqrt{\left\langle\left|H_{+}\right|^{2}\right\rangle\left\langle\left|H_{-}\right|^{2}\right\rangle}},
$$

in the range $4.5-5.5 \mathrm{mHz}$ for the quiet subphotosphere at a depth of $7 \mathrm{Mm}$. Figure $8 a$ shows the normalized control correlation, $\hat{C}_{\mathrm{LC} \pm}$, in the $4.5-5.5 \mathrm{mHz}$ range for comparison, taken from Figure $5 a$. Figure $8 b$ shows the egression-ingression correlation with the acoustic progressions computed for the normalized pressure, $\dot{\eta}^{3}{ }^{3}$ The difference between the two is considerable. This leads us to a recognition of analogs of the "ghost signatures" introduced in $\S 5.3$, which now contribute noise that is not only quite significant but also systematic.

\subsection{The Ghosts}

If we could compute only the correlation

$$
C_{\uparrow \downarrow}(\boldsymbol{r}, z) \equiv\left\langle H_{\uparrow+}(\boldsymbol{r}, z, \omega) H_{\downarrow-}^{*}(\boldsymbol{r}, z, \omega)\right\rangle_{\Delta \omega},
$$

we would expect little systematic difference between $C$ and the control correlations, $C_{\mathrm{LC} \pm}$ (outside of a phase shift to account for phase differences between $\eta$ and $\zeta$ ). In fact, the full ingression-egression correlation, $C$, contains analogs of the ghost signatures introduced in $\S 5.3$ (see eq. [58]). It is the sum of $C_{\uparrow \downarrow}$ and three cross terms:

$$
C=C_{\uparrow \downarrow}+C_{\downarrow \downarrow}+C_{\uparrow \uparrow}+C_{\downarrow \uparrow},
$$

where we generalize $C_{\uparrow \downarrow}$ defined by equation (69) by a formalism analogous to that expressed by equation (59) for the control correlations:

$$
C_{\mu \nu}(\boldsymbol{r}, z) \equiv\left\langle H_{\mu+}(\boldsymbol{r}, z, \omega) H_{\nu-}^{*}(\boldsymbol{r}, z, \omega)\right\rangle_{\Delta \omega},
$$

with $\mu, \nu \in\{\uparrow, \downarrow\}$. As in their analogs in the control correlations (see eqs. [58] and [59]), it is the unwelcome cross terms, $C_{\downarrow \downarrow}, C_{\uparrow \uparrow}$, and $C_{\downarrow \uparrow}$, that we call the ghost signatures. These components interfere with $C_{\uparrow \downarrow}$ to produce the deformedpaperclip-like locus plotted in Figure $8 b$.

The ghost signatures projected by individual shallow, horizontally localized acoustic sources defocus as the focal plane submerges, spreading out over a large area and therefore fading rapidly. However, integrated over a large area, as are the statistics plotted in Figure 8, the ghosts can persist to a significant depth at any single frequency.

The components $C_{\uparrow \uparrow}$ and $C_{\downarrow \downarrow}$ represent the ghost contributions to ingression and egression computations in a common pupil of the same noise field, as opposed to one that is an echo of another from a lower turning point. These can be simulated simply by computing the ingression of a random

\footnotetext{
${ }^{3}$ Because vertical acoustic motion at the surface translates to primarily horizontal motion at a lateral focal point, the acoustic projections for $C$ plotted in Figs. $8 b$ and $8 d$ were computed for the time derivative of normalized pressure, $\dot{\eta}$, not vertical displacement, $\dot{\zeta}$. No correction is applied to account for the phase shift between $\eta$ and $\zeta$ in Figs. $8 b$ and $8 d$.
}
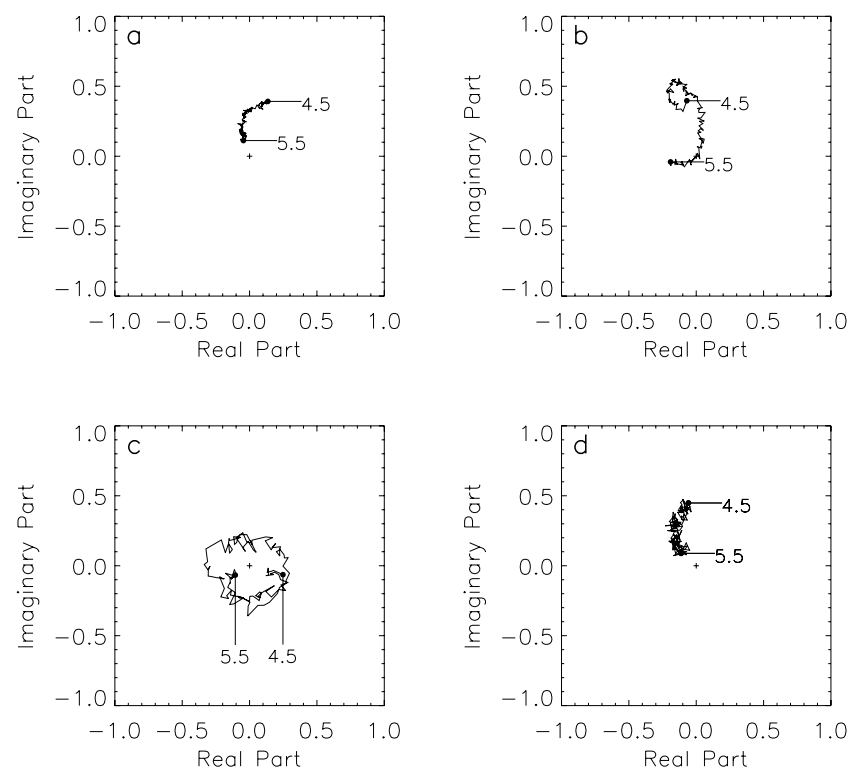

Fig. 8.- Normalized correlation amplitude statistics over the frequency range $4.5-5.5 \mathrm{mHz}$. Panel $(a)$ shows the normalized control correlation, $\hat{C}_{\mathrm{LC}-}$ (see eq. [51]), for the quiet Sun (also plotted in Fig. 5a). Panel (b) shows the normalized egression-ingression correlation, $\hat{C}$, (see eq. [68]) with both ingression and egression computed in the lateral vantage over a common same annular self-conjugate pupil with the focal point $7 \mathrm{Mm}$ beneath the quiet photosphere. Panel (c) shows the same computation for simulated noise, to draw out the ghost component $C_{\uparrow \uparrow-}+C_{\downarrow \downarrow-}$. Panel $(d)$ shows $\hat{C}$ with ingression and egression computed in the lateral vantage over mutually conjugate quarterannular pupils separated in the east-west direction. Statistics are poorer because of the reduced pupils. However, the ghost signatures are greatly reduced, resulting in a locus whose shape is similar to that of the control correlations.

noise field with no acoustic echo. Figure $8 c$ demonstrates this by plotting $C$ for acoustic projections applied to simple, numerically created random Gaussian noise whose spatial spectrum is uniform out to the value of $k$ for the fundamental mode, where it is sharply truncated. The phases of $C_{\uparrow \uparrow}$ and $C_{\downarrow \downarrow}$ tend to progress at a rate $d \phi / d \omega \sim T$, where $T$ can be regarded as the travel time, averaged over the pupil, of acoustic radiation from the solar surface to the lower turning point and back. It should be clear that the expectations of both $C_{\uparrow \uparrow}$ and $C_{\downarrow \downarrow}$ will be essentially null when the ingression and egression pupils are separate and disjoint.

The third ghost, $C_{\downarrow \uparrow}$, is the result of progressions applied to real acoustic noise that does have an echo but is traveling in the direction opposite to that of the disturbances on which $C_{\uparrow \downarrow}$ is focused. Elementary considerations in optics can be used to show that if the ingression and egression pupils are substantial in extent but well separated, then $C_{\downarrow \uparrow}$, like $C_{\uparrow \uparrow}$ and $C_{\downarrow \downarrow}$, is largely neutralized. As a result, when the ingression and egression are computed over mutually conjugate quarter-annular pupils (see Fig. $8 d$ ), the locus of $C$, while relatively noisier as a result of considerably reduced statistics, recovers a shape similar to that of the control correlations. This is not strictly the case for phase-correlation statistics beneath a strong magnetic region.

\section{PHASE-CORRELATION HOLOGRAPHY OF AN ACTIVE REGION}

Figure 9 shows subphotospheric phase-correlation maps of NOAA AR 8179 (magnetic complex centered in gray-tone panels) and 8178 (isolated sunspot above and left of panel center) on 1998 March 15 integrated over a $10.6 \mathrm{hr}$ interval beginning at 11:00 UT with the focal plane at a depth of 4.2 $\mathrm{Mm}$. The top two panels show the visible continuum (a) 


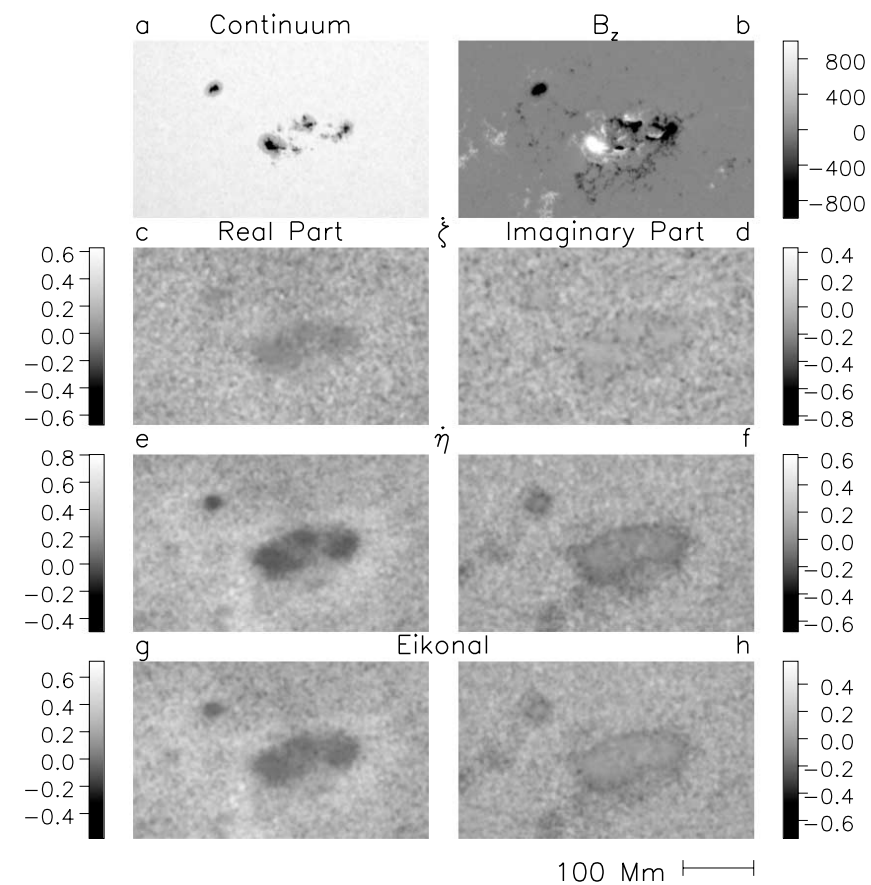

FIG. 9.-Maps of egression-ingression correlation, $C, 4.2 \mathrm{Mm}$ beneath NOAA AR 8179 integrated over the $4.5-5.5 \mathrm{mHz}$ spectrum. Panel $(a)$ shows an MDI white-light image of the active region. Panel $(b)$ shows the vertical component, $B_{z}$, of the magnetic field. Panels $(c)$ and $(d)$ show the real and imaginary parts, respectively, of $C$ for hydromechanical progressions of the normalized vertical velocity, $\dot{\zeta}$. Panels $(e)$ and $(f)$ show the same for the time derivative of normalized pressure, $\dot{\eta}$. Panels $(g)$ and $(h)$ show the correlation amplitude for eikonal progressions. The average value of $C$ for the quiet Sun has been subtracted from all of the phase-correlation maps for an optimal representation of the signature of the active region with respect to the quiet Sun.

and the vertical component of the magnetic field, $B_{z}(b)$, computed from the MDI magnetogram of 1998 March 15, 16:00 UT, assuming that the magnetic vector, $\boldsymbol{B}$, is the gradient of a potential. Hydromechanical acoustic regressions, $H_{+}$, and progressions, $H_{-}$, for normalized displacement, $\zeta$, and normalized pressure, $\eta$, were applied to the observations, and respective phase correlations, $C$, integrated over the $4.5-$ $5.5 \mathrm{mHz}$ spectrum as prescribed by equation (9). The acoustic projections were phase-corrected for dispersion as described in the last paragraph of $\S 5.3$. The resulting phase correlation maps are shown in the second and third rows of the figure. Panels at left and right show the real and imaginary parts of $C$, respectively, with $\psi$ equated to $\dot{\zeta}$. The hydromechanical regressions therefore represent $\dot{\zeta}$ (second row) and $\dot{\eta}$ (third row). $C$ is also shown for progressions computed under the eikonal formalism (bottom row, see $\S 3$ ).

The difference between the correlation signatures of vertical displacement and pressure is considerable, the granular texture of the former being considerably coarser. The reason for this is that the waves with highest $l$ are those whose turning points are at or above the focal plane, and the kinetic energy of the wave mode at the turning point is invested primarily in horizontal motion at the expense of vertical. This consideration can be assessed in quantitative terms by comparing the real parts, $U_{r}$ and $V_{r}$, of $U^{ \pm}$and $V^{ \pm}$as plotted in Figures 2 and 3. Well above the turning point, $U_{r}$ and $V_{r}$ range over comparable limits in amplitude. In the neighborhood of the turning point and below, $\left|V_{r}\right|$ is generally much greater than $\left|U_{r}\right|$. When the correlation is computed, the suppression ratio is effectively squared. The signature in $\dot{\zeta}$ is therefore more representative of the lower $l$ waves, whose turning points are some distance beneath the focal plane. By comparison, the pressure signature, $\dot{\eta}$, represents the entirety of the compressional energy at the turning point as elsewhere. It therefore represents the high- $l$ spectrum much more in proportion to the total energy that penetrates to the focal plane.

The similarity between the eikonal correlation map and that of normalized pressure, $\dot{\eta}$, in Figure 9 is as conspicuous as the difference between the latter and that of the normalized vertical displacement, $\dot{\zeta}$. Figure 10 shows statistical plots comparing moduli (Fig. 10a) and phases (Fig. 10b) of individual hydromechanical and eikonal pixels at $4.2 \mathrm{Mm}$ depth. The correlations are high even for individual pixels at the diffraction limit. When corrected for a factor of 2.5 by which the moduli differ
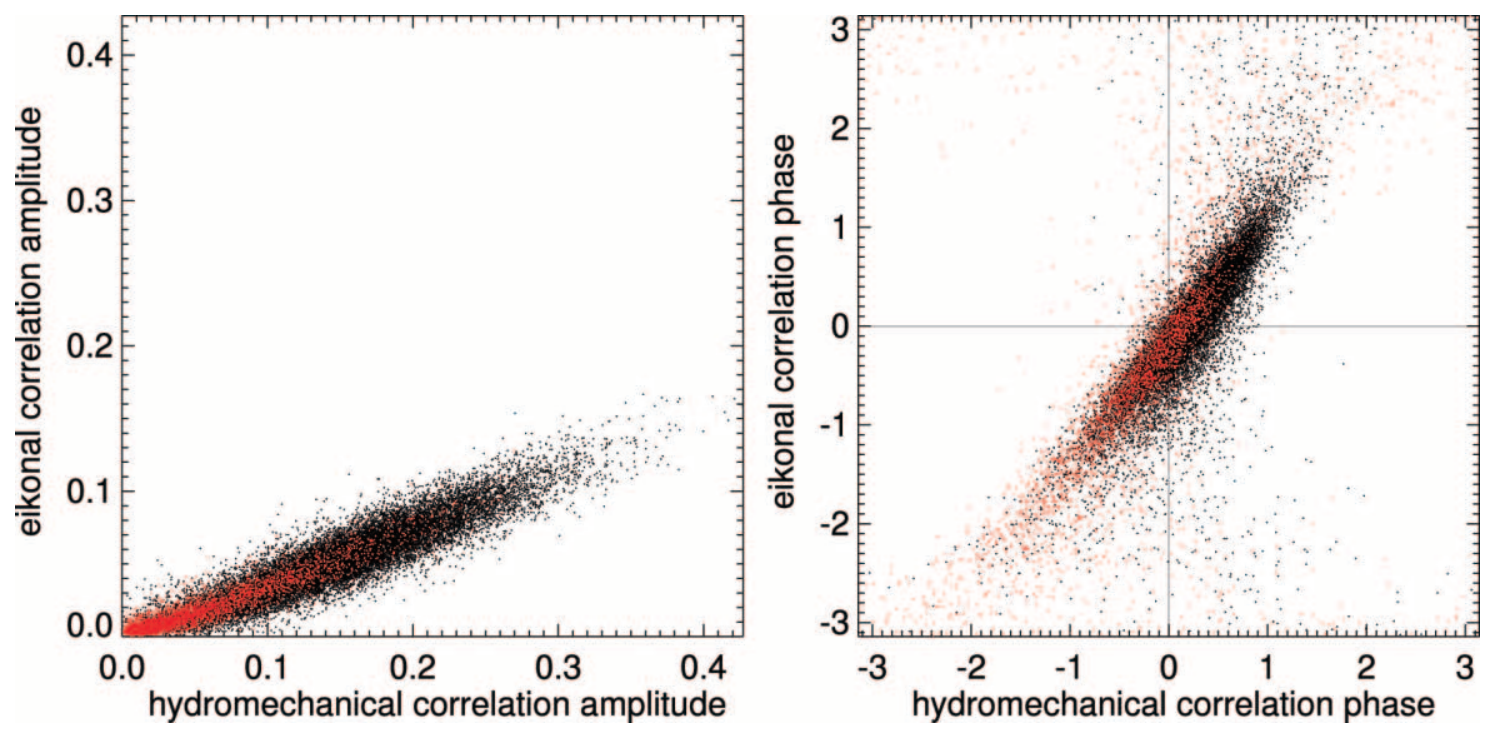

FIG. 10.-Egression-ingression correlation values, $C$, are plotted for hydromechanical progressions of the time derivative of pressure (horizontal axes) and

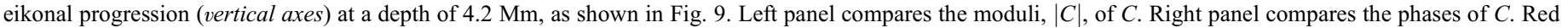

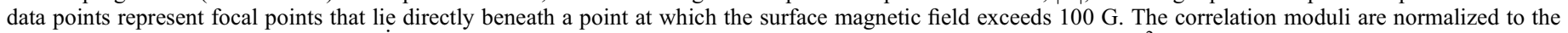
mean $4.5-5.5 \mathrm{mHz}$ acoustic power in $\dot{\zeta}$ in the quiet Sun as registered by the MDI observations, which is $2.1 \mathrm{~kW} \mathrm{~m}^{-2}$ at the base of the photosphere. 


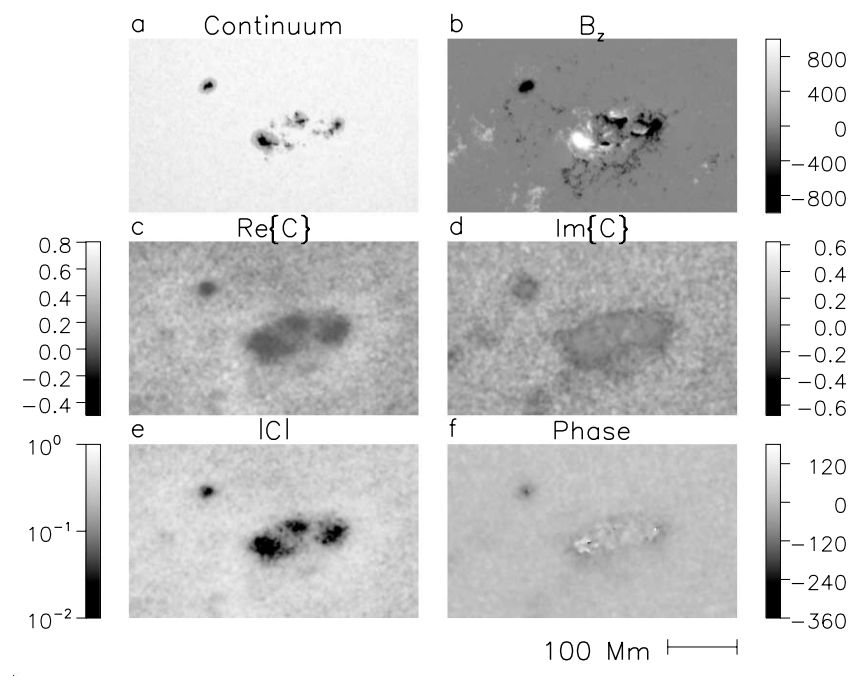

FIG. 11.-Real and imaginary parts of the complex correlation amplitude, $C$, of NOAA AR 8179 at a focal-plane depth of $4.2 \mathrm{Mm}$, integrated over the 4.5-5.5 mHz spectrum, are compared with the modulus and phase of $C$. The acoustic progressions in this figure represent the time derivative of the normalized pressure, $\dot{\eta}$. The top row shows white-light and vertical magnetic field images (same as top row of Fig. 9). The second row shows the real and imaginary parts of $C$ (same as third row of Fig. 9). Panel (e) shows of the modulus of $C$, rendered logarithmically. Panel $(f)$ shows the phase of $C$.

and a phase difference of 5.8 between the two, the eikonal approximation renders an excellent facsimile of the hydromechanical computations in the $4.5-5.5 \mathrm{mHz}$ spectrum for a depth of $4.2 \mathrm{Mm}$ or below. A careful comparison between active region (red pixels) and quiet Sun (black) shows that the basic relationship between hydromechanical and eikonal correlations is substantially independent of the magnetic field strength in the pupil.

In view of the more proportionate representation of the high- $l$ acoustic spectrum and the finer spatial resolution it offers, we will focus our attention from this point on correlation amplitudes for the normalized pressure, $\dot{\eta}$. Figure 11 shows comparative images of the real and imaginary parts of the correlation amplitude and its modulus and phase. As in Figure 9, the top row shows the visible continuum (left) and the vertical component, $B_{z}$, of the magnetic field (right). The second row shows the real (left) and imaginary (right) parts of $C$, as in the third row of Figure 9. The bottom row shows the modulus (left, rendered logarithmically) and the phase (right) of $C$, for which a negative (dark) perturbation is equivalent to a reduced travel time from the surface through the focus and back. The modulus of $C$ in the active region is only a small fraction of that of the quiet Sun. In general, the photospheric Doppler signature of acoustic waves impinging from the solar interior into regions whose surface magnetic fields are stronger than about $250 \mathrm{G}$ is considerably smaller than in the quiet Sun. This complicates the discrimination of detail near the diffraction limit in the shallow subphotospheres of active regions. For the moment we will concern ourselves with only the relatively large-scale phase variation of $C$, leaving the effects of the surface magnetic fields uncorrected.

Figure 12 shows lateral-vantage phase maps of $\phi^{S}$ for a single self-conjugate pupil, which in this case is simply $\arg C$, for AR 8179 over focal plane depths ranging from 1.4 to 8.4 Mm. The left column shows the signature of the active region. The panel to the right of each phase map in Figure 12 contains a map of the real part of the Green's function applied

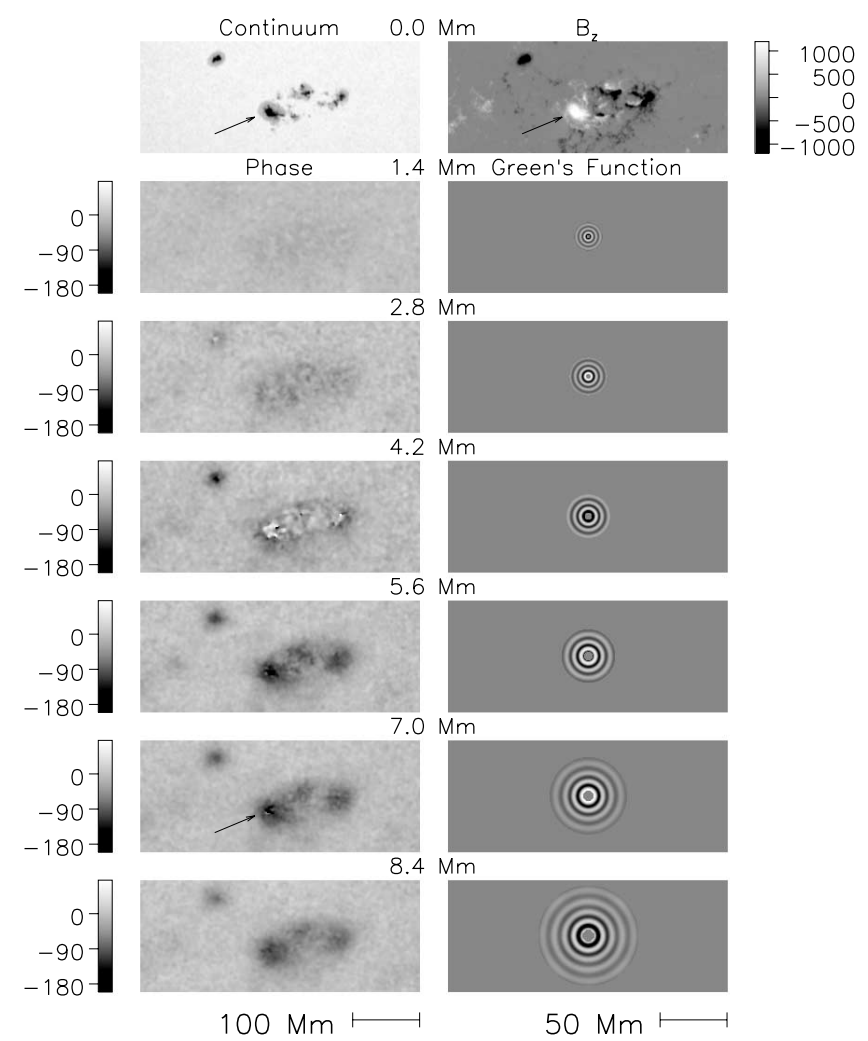

FIG. 12.-Lateral-vantage maps of phase, $\phi^{S}$, of $C$ over the $4.5-5.5 \mathrm{mHz}$ spectrum with $H_{ \pm}$computed over a self-conjugate annular pupil are rendered in gray tones at depths ranging from 1.4 to $8.4 \mathrm{Mm}$ beneath the photosphere of NOAA AR 8179. The top row shows white-light (left) and vertical magnetic (right) images of the region. Underlying panels in left column show $\phi^{S}$ at depths expressed above respective upper right corners. The panel to the right of each phase map shows the real part of the Green's function, truncated by the lateral-vantage pupil. The scale on which the Green's functions are rendered is magnified with respect to that of the phase maps by a factor of 2 .

to the surface acoustic field to focus it to the depth indicated above. These "point-source holograms" are characterized by a pattern of circular fringes truncated by the pupil annulus. Because the fringes of the shallower holograms are a little bit too fine to print on the scale of the phase maps, they are rendered here at twice the magnification of the latter.

Figure 13 shows maps of the symmetric phase, $\phi^{S}$, for AR 8179 (see eq. [66]), for which the ingression and egression pupils are separate quarter annuli, lying to the east and west of the focal point. The gray-tone panels in the left column show the phase maps. The gray-tone panels in the right column show respective Green's functions truncated by the union of the east and west pupils. Figures 12 and 13 show significant differences as well as some fairly conspicuous similarities. These will be the subject of some of the discussion in the next section.

\section{DISCUSSION}

\subsection{Accuracy of the Eikonal Approximation}

Barnes \& Cally (2001) expressed concerns as to the validity of the eikonal approximation for diagnostic applications in the shallow subphotosphere where dispersion is significant. The formalism we have applied heretofore (Lindsey \& Braun 1997, 2000 b) to correct the eikonal approximation for dispersion is relatively crude, based on empirical statistics that neglect the dependence of dispersion on $l$, which Barnes \& Cally (2001) showed to be significant at relatively low frequencies in the 


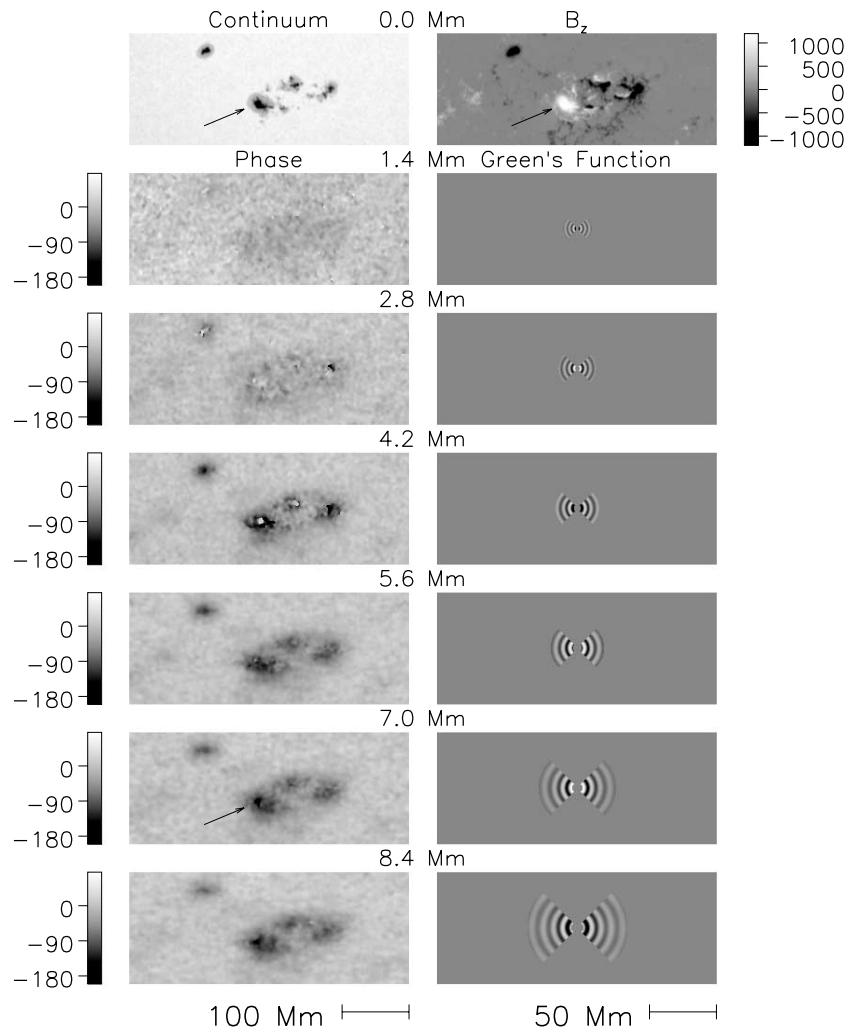

FIG. 13.-Symmetric phase, $\phi^{S}$ of the of the $4.5-5.5 \mathrm{mHz}$ ingressionegression correlations for separate, mutually conjugate ingression and egression pupils is rendered as in Fig. 12. The pupils are quarter-annuli identical in inner and outer radii to respective pupils applied in Fig. 12, separated in the east-west direction. The panel to the right of each phase map shows the real part of the Green's function, truncated by the union of the ingression and egression pupils. The scale on which the Green's functions are rendered is magnified with respect to that of the phase maps by a factor of 2 .

shallow subphotosphere. The concerns raised by Barnes \& Cally (2001) were based on computations of wave-packet trajectories, which deviate somewhat more from the eikonal trajectories than do phase trajectories, which follow the gradient of the phase of $G_{ \pm}$(see eqs. [10] and [11]). As Barnes \& Cally (2001) themselves mentioned, the basis of holographic reconstruction is more a matter of phase coherence than of wave-packet trajectories. The trajectories plotted by Barnes \& Cally (2001) deviate little more than a $\mathrm{Mm}(\sim 0.7$ pixel) from the eikonal for frequencies in the range $4.5-5.5 \mathrm{mHz}$ with focal plane depths of order $5 \mathrm{Mm}$. It should therefore not be surprising that the eikonal approximation renders phase correlation maps very close to those derived from the hydromechanical reconstruction of pressure for depths in the neighborhood of $5 \mathrm{Mm}$ and frequencies above $4.5 \mathrm{mHz}$. For lower frequencies and shallower focal planes, the eikonal approximation is open to more significant errors. Our comparisons suggest that the eikonal approximation is safe when the eikonal travel time from the focal plane to the photosphere is greater than approximately $1.5 / \nu$. Pending a more extensive study, the most reliable answer to the concerns cited by Barnes \& Cally (2001) is hydromechanical computations in place of the eikonal for eikonal travel times less than $1.5 / \nu$.

\subsection{The Large-Scale Active Region Signature}

The phase signatures of AR 8179 beneath $5.6 \mathrm{Mm}$ in Figures 12 and 13 can be characterized predominantly as a defocused representation of the surface magnetic regions as the focal plane submerges. The signature reaches a maximum directly beneath the large sunspot on the east side of AR 8179 . The maximum is 2.5 radian at $5.6 \mathrm{Mm}$ and gradually decrease with depth, to 1.7 radian at $8.6 \mathrm{Mm}$. These signatures convey phase advances, equivalent to travel time reductions of $80 \mathrm{~s}$ and $54 \mathrm{~s}$, respectively, for $5 \mathrm{mHz}$ radiation. Phase-correlation holography applied to superficial model anomalies by Lindsey \& Braun (2004a, 2004b) support the proposition that the phase signatures seen at $5.6 \mathrm{Mm}$ and below in Figures 12 and 13 are predominantly the result of an acoustic anomaly that is relatively superficial. For modeling purposes, such an anomaly can be roughly characterized by the removal of the upper $\sim 350 \mathrm{~km}$ of the solar subphotosphere, or equivalently depressing the solar surface so that the wave arrives at the photosphere substantially ahead of schedule as compared to the nonmagnetic subphotosphere (Lindsey \& Braun 2004b). By removing the upper $350 \mathrm{~km}$ from the model subphotosphere of ChristensenDalsgaard et al. (1993), for example, we reduce the one-way travel time by $40 \mathrm{~s}$, for a two-way reduction that matches the phase signature of AR 8179 at $5.6 \mathrm{Mm}$.

We will refer to the superficial part of the anomaly that causes the large-scale diffuse signature by the term "acoustic Wilson depression." It should be understood that, while magnetic forces are known to depress the solar photosphere visibly (Bray \& Loughead 1964), shortening the ray path and thereby reducing the travel time, the actual mechanism of the phase shift caused by magnetic fields is considerably more complicated than a simple removal of the upper few hundred $\mathrm{km}$ of the subphotosphere. There is good reason to suppose that the large-scale diffuse signature is at least partly the result of a change in the amplitude of a photospheric or subphotospheric reflection, or the depth from which such a reflection occurs (Braun \& Lindsey 2000b). Other likely contributors include magnetic forces, any thermal anomaly too shallow to be resolved acoustically, a local variation in the parameter $z_{S}$ (see eq. [64]) that characterizes the range over which acoustic waves are generated, and other qualities of the shallow magnetic subphotosphere that differ acoustically from those of the quiet subphotosphere. There are arguments grounded in elementary MHD, for example, to suggest that relatively modest photospheric magnetic fields can introduce local phase shifts up to a radian (Cally et al. 2003), apart from a physical displacement of the medium.

Until the acoustics of the shallow magnetic subphotosphere are better understood, and a diagnostic basis for distinguishing different superficial anomalies from one another is secured, we will use the term "acoustic Wilson depression" to encompass the generality of physical mechanisms, and prospective superficial anomalies based thereon, that could give rise to the largescale diffuse phase-correlation signature. It should therefore be understood that we will now use this term in a more general sense than before, for example, in Braun \& Lindsey (2000b).

\subsection{The Ghosts, Revisited Thereby}

The phase signature of AR 7179 at depth $4.2 \mathrm{Mm}$ in Figure 12 shows sharply defined "islands" of positive phase directly beneath regions of relatively strong $B_{z}$. We attribute these islands largely to the ghosts, introduced in $\S 5$.6. In the case of Figure 12, this attribution is based heavily on the considerable weakening of these apparitions when the ingression and egression pupils are separated (see Fig. 13). The ghost contributions of compact anomalies fade rapidly with increasing depth as a result of defocusing. As we mentioned in $\S 5.6$, by computing $H_{+}$and $H_{-}$over disjoint pupils, $C_{\uparrow \uparrow}$ and 
$C_{\downarrow \downarrow}$ are eliminated. For the well-separated quarter-annular pupils, the ghost signature $C_{\downarrow \uparrow}$ is a small fraction of the desired $C_{\uparrow \downarrow}$ correlation for a focus substantially submerged beneath the quiet photosphere. However, according to our present understanding, large phase perturbations due to strong photospheric magnetic fields can reintroduce significant contamination of $C$ by $C_{\downarrow \uparrow}$, among other complications, even when the ingression and egression pupils are substantially separated. Under these conditions there are good reasons to at least suspect that the relative contribution from $C_{\uparrow \downarrow}$, and interference therefrom, could be a significant contributor to the somewhat reduced but still conspicuous island-like signatures seen in Figure 13. All of the sharply defined signatures in Figure 13, including the deep one indicated by an arrow at $7 \mathrm{Mm}$, occur in regions where $|\hat{C}|$ is severely reduced, as in Figure $11 e$, where interference from a relatively small perturbation on $C$ can exert a violent phase shift. Implications of the ghost signatures in active region acoustics are addressed in somewhat more detail by Lindsey \& Braun (2004b).

\subsection{The Acoustic Showerglass}

At this point we confront a major problem that looms over local helioseismic diagnostics of the shallow subphotospheres of active regions in some generality, particularly at high frequencies. It is now evident that the strong acoustic anomalies that pervade the upper few hundred $\mathrm{km}$ of the subphotosphere introduce large phase perturbations that significantly impair the coherence of acoustic radiation propagating into them from beneath. The phase maps rendered in Figures 12 and 13 indicate one-way phase shifts exceeding 3 radians from the quiet Sun to the centers of large sunspots. It is rapidly becoming evident (Lindsey \& Braun 2003, 2004a, 2004b) that most of these phase perturbations occur within a few hundred $\mathrm{km}$ of the surface. This means not only that acoustic anomalies significantly beneath active region photospheres are dwarfed by those that lie above them, but that the Born approximation is substantially violated by the surface perturbation alone. For purposes of acoustics, the active region photosphere can be likened to the function of the familiar "showerglass" in electromagnetic optics. The assessment and correction of the acoustic showerglass is a substantial undertaking, currently under development, that runs somewhat beyond the practical scope of this paper. This is the subject of a forthcoming publication (Lindsey \& Braun 2004a, 2004b) specifically focused on this problem in acoustic diagnostics of active region subphotospheres.

\section{SUMMARY}

We have worked out the general "hydromechanical" formalism for computational seismic holography in the planeparallel approximation according to standard acoustic models of the solar interior. We have applied the hydromechanical formalism for phase-correlation holography to helioseismic observations of an active region and favorably compared the resulting signatures to those computed under the "eikonal approximation," under which helioseismic holography was substantially developed. Comparisons between holographic images computed according to the eikonal formalism of Lindsey \& Braun $(1997,2000 \mathrm{~b})$ and those based on the hydromechanical formalism show that eikonal reconstruction with a fairly simple correction for dispersion serves as an excellent approximation to hydromechanical acoustics for a broad range of diagnostic applications.

The phase correlation between ingression and egression computations generally contains artifacts we call "ghost signatures." These are largely the result of ambiguity in the travel direction of waves based on the surface disturbance recorded in standard helioseismic observations. The ghost signatures can be largely eliminated in the quiet subphotosphere by keeping the ingression and egression pupils well separated, provided the individual pupils are sufficient in radial extent. This is not entirely the case for acoustic diagnostics of a magnetic subphotosphere.

The phase correlation signatures of the shallow subphotospheres of active regions are predominated by large amplitude and phase perturbations imposed by surface magnetic fields. These act as an "acoustic showerglass," significantly impairing the coherence of acoustic radiation from subsurface acoustic anomalies. Intelligible diagnostics of the shallow subphotospheres of active regions require a particularly careful account of the showerglass effect.

We dedicate this work to the memory of Karen Harvey (1942-2002), our sponsor, respected colleague, a true friend, and always a great inspiration. We most appreciate the continual encouragement and support of Jack Harvey at the National Solar Observatory and the Solar Physics Research Corporation. A major part of the work reported in this paper was done during the tenure of one of the authors, C. L., at the Solar Physics Research Corporation, in Tucson, Arizona. Discussions with Aaron Birch contributed greatly to our understanding of various acoustic considerations that influence holographic phase correlation signatures. We thank the anonymous referee for a particularly conscientious and insightful review. This work was supported by grants from the Supporting Research and Technology Program and the Living with a Star Program of the National Aeronautics and Space Administration and from the Stellar Astronomy and Astrophysics Program of the National Science Foundation.
Barnes, G., \& Cally, P. S. 2001, Publ. Astron. Soc. Australia, 18, 243 Berkhout, A. J. 1985, Seismic Migration (Oxford: Elsevier)

Born, M., \& Wolf, E. 1975, Principles of Optics (Oxford: Pergamon), 109

Braun, D. C., \& Lindsey, C. 1999, ApJ, 513, L79

2000a, Sol. Phys., 192, 285

2000b, Sol. Phys., 192, 307

2001, ApJ, 560, L189

Braun, D. C., Lindsey, C., Fan, Y., \& Fagan, M. 1998, ApJ, 502, 968

Bray, R. J., \& Loughead, R. E. 1964, Sunspots (New York: Dover)

Cally, P. S., Crouch, A. D., \& Braun, D. C. 2003, MNRAS, 346, 381

Castle, Q. Q. 1982, Geophysics, 47, 1001

Christensen-Dalsgaard, J., Proffitt, C. R., \& Thompson, M. J. 1993, ApJ, 403, L75

Claerbout, J. F. 1970, Geophysics, 35, 407

\section{REFERENCES}

Claerbout, J. F. 1971, Geophys. J. RAS, 26, 285

Claerbout, J. F., \& Doherty, S. M. 1972, Geophysics, 37, 741

Donea, A.-C., Braun, D. C., \& Lindsey, C. 2000, Sol. Phys., 192, 321

Donea, A.-C., Lindsey, C., \& Braun, D. C. 1999, ApJ, 513, L143

Duvall, T. L. Jr., Jefferies, S. M., Harvey, J. W., \& Pomerantz, M. A. 1993, Nature, 362, 430

Hilterman, F. J. 1970, Geophysics, 35, 1020

Kumar, P. 1994, ApJ, 428, 827

Kumar, P., \& Basu, S. 2000, ApJ, 545, L65

Lindsey, C., \& Braun, D. C. 1990, Sol. Phys., 126, 101

- 1997, ApJ, 485, 895

1998, ApJ, 499, L99

1999, ApJ, 510, 494

2000a, Science, 287, 1799 
Lindsey, C., \& Braun, D. C. 2000b, Sol. Phys., 192, 261 2003, in Proc. SOHO 12/GONG+ 2002, Local and Global Helioseismology: The Present and Future, ed. H. Sawaya-Lacoste (Nordwijk: ESA), 23

2004a, ApJ, in press

$2004 \mathrm{~b}$, ApJ, in press

Musgrave, A. 1961, Geophysics, 26, 738
Roddier, F. 1975, Comput. Rend. Acad. Sci., 281, B993

Schneider, W. A. 1978, Geophysics, 43, 49

Skartlien, R. 2001, ApJ, 554, 488 2002, ApJ, 565, 1348

Thomas, J. H., Clark, P. A., \& Clark, A. Jr. 1971, Sol. Phys., 16, 51

Unno, W., Osaki, Y., Ando, H., Saio, H., \& Shibahashi, H. 1989, Nonradial Oscillations of Stars (Tokyo: Univ. Tokyo Press), 108 